\title{
Lixiviats Issus De La Decharge Publique De La Ville De Meknes : Caracterisation Et Impact Sur Les Eaux De L'oued Boufekrane
}

\author{
Lekehal Hicham .Dr, \\ Bouchelta Aziz. Ph \\ Université Moulay Ismail, Faculté des Sciences, département de Biologie, \\ Laboratoire de recherche Environnement \& \\ Santé Dép. Biologie, Zitoune Meknès, Maroc. \\ Benzougagh Brahim. Dr \\ Université Moulay Ismail, Faculté des sciences Département de Géologie, \\ Laboratoire de Géoscience, Patrimoine et substances utiles (GPS), \\ Zitoune, Meknès- Maroc \\ Boukhari Taleb Roukia. Dr \\ Institut Agronomique et Vétérinaire Hassan II (IAV), Unité de Recherche : \\ Gestion conservatoire des eaux et des sols, Rabat, Maroc
}

Doi:10.19044/esj.2018.v14n29p332 URL:http://dx.doi.org/10.19044/esj.2018.v14n29p332

\begin{abstract}
Leachate from the public landfill of the city of Meknes is considered as a source of significant pollution of the waters of the Boufekrane wadi and agricultural land bordering the landfill. The objective of this study is the characterization of the physicochemical parameters of the leachates as well as the determination of the degree of pollution of the local superficial waters in order to propose adequate techno-economic solutions to remedy this problem. The results obtained following the characterization of the leachates showed that they represent a very important salinity (electrical conductivity: $30805 \mu \mathrm{s}$ $/ \mathrm{cm}$ ) with a strong organic and mineral pollutant load (BOD5: 598mg / 1, COD: 3346mg / 1 and Chlorides: 5144mg / 1) .Water quality monitoring in Boufekrane Oued has shown that the qualities are poor and that the contents of the majority of the parameters analyzed are well above the limit values for direct discharges into the receiving environment (EC: $9642 \mu \mathrm{s} / \mathrm{cm}, \mathrm{COD}$ : 910mg / 1, BOD5: 320mg / 1, dissolved oxygen: 7.2mg / 1, Chlorides: 4470mg /1, Sulphates: $435 \mathrm{mg} / 1$ ). The principal component analysis confirmed that the results obtained are very consistent and consistent with the descriptive analysis.
\end{abstract}

Keywords: Leachates, dumps, water, pollution, infiltration, Meknes. 


\section{Résumé}

Les lixiviats issus de la décharge publique de la ville de Meknès sont considérés comme source de pollution importante des eaux de l'oued Boufekrane et des terrains agricoles limitrophes à la décharge. L'objectif de cette étude est La caractérisation des paramètres physico-chimiques des lixiviats ainsi que la détermination du degré de pollution des eaux superficielles de proximité afin de proposer des solutions technicoéconomiques adéquates pour remédier à ce problème.Les résultats obtenues suites à la caractérisation des lixiviats ont montré que ces derniers représentent une salinité très importante(conductivité électrique : $30805 \mu \mathrm{s} / \mathrm{cm}$ ) avec une forte charge polluante organique et minérale(DBO5 : 598mg/l, DCO : 3346mg/l et Chlorures :5144mg/l).Le suivi de la qualité des eaux de l'Oued Boufekrane à montré quelle sont de mauvaises qualités et que les teneurs de la majorité des paramètres analysés sont largement supérieures aux valeurs limites des rejets direct dans le milieu récepteur(CE : 9642 $\mu \mathrm{s} / \mathrm{cm}$, DCO : 910mg/1, DBO5 : 320mg/l, Oxygène dissous : 7,2mg/1, Chlorures :4470mg/1, Sulfates : 435mg/l). L'analyse en composante principale a confirmé que les résultats obtenus sont très cohérents et concorde avec l'analyse descriptive.

Mots Clés : Lixiviats, décharge publique, eau, pollution, paramètres physicochimiques, Oued Boufekrane, Meknès.

\section{Introduction:}

Les lixiviats sont des effluents toxiques issus des décharges ou centres de stockages des déchets, ils résultent de la percolation de l'eau à travers le massif des déchets, de l'eau contenue dans les déchets et de celle apportée par les précipitations .Ils sont composés de multiples éléments organiques et minéraux et peuvent contaminer les nappes phréatiques, les eaux superficielles et les sols s'ils ne sont pas récupérés et traités avant leurs rejets dans le milieu naturel (Chofqi et al., 2007).

Après avoir estimé le débit des lixiviats issus de la décharge publique de la ville de Meknès, dans le cadre d'une étude antérieure en 2016 réalisée par notre équipe de recherche, recommandant ainsi d'effectuer une caractérisation physico-chimique et bactériologique des lixiviats afin de proposer des solutions technico-économiques pour remédier à ce problème. La présente étude se focalisera seulement sur la description des principales caractéristiques physiques et chimiques de ces lixiviats ainsi que celles des eaux de l'oued Boufekrane. Le suivi de leur évolution saisonnière et la compréhension des mécanismes fondamentaux qui les régissent.

Pour ce faire, nous avons effectué un suivi spatio-temporel des paramètres suivants : température de l'eau, $\mathrm{pH}$, conductivité, nitrates, demande 
biologique en oxygène après 5 jours, demande chimique en oxygène, matières en suspension et oxygène dissous.

\section{Présentation de la zone d'étude}

La décharge de la ville de Meknès dont le projet d'aménagement et réhabilitation en cours de réalisation desserve la totalité de la zone urbaine de Meknès. Elle a été mise en service en l'an 2002. La quantité des déchets arrivant à la décharge est estimée à 554 tonnes par jour en 2015 avec un taux moyen d'humidité de $40 \%$. Le site, d'une superficie de 17 hectares, reçoit une pluie efficace moyenne de $475 \mathrm{~mm} / \mathrm{an}$, générant ainsi un volume total estimé à $271 \mathrm{~m}^{3} / \mathrm{j}$ de lixiviats. L'impact de ces derniers sur les eaux superficielles est fort, puisque les lixiviats qui ne peuvent pas s'infiltrer dans le sous-sol, ruissellent, selon une ligne d'écoulement et impactent directement l'oued Boufekrane après avoir traversé des zones de cultures et de pâtures.

\section{Contexte géologique}

Il occupe la partie sud-ouest du bassin de Saïss (fig.01). Il a une forme semi-triangulaire avec une surface deux fois plus grande que son homologue nord-oriental. Sa plus grande dimension est d'environ $60 \mathrm{~km}$ d'ouest en est, s'accompagne également d'un relèvement assez remarquable matérialisé par un doux étagement du plateau depuis les $500 \mathrm{~m}$ de la localité de Aïn Orma à l'ouest, jusqu'aux 700m (fig.02) à la pointe du Bitit (Fassi, 1999). La différenciation de la pente entre le Nord et le Sud, influence la morphologie des cours d'eau et la vitesse de circulation des eaux souterraines (Amraoui, 2005).

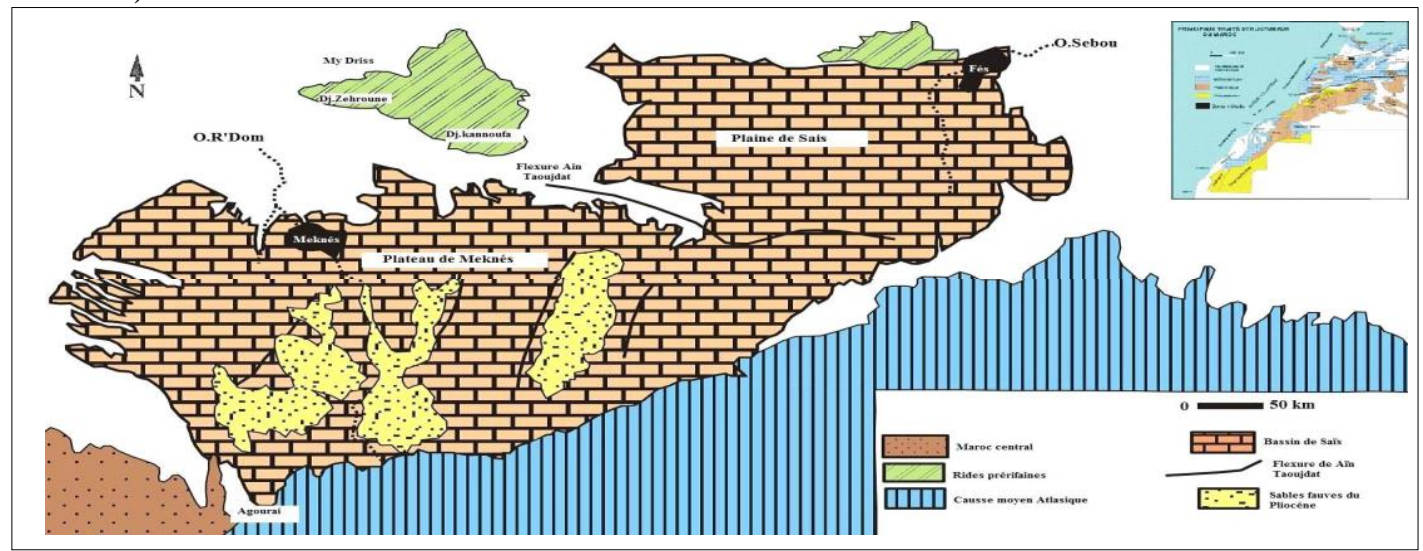

Figure 01 : Esquisse géologique du bassin de Saïss (Taltasse, 1953). 


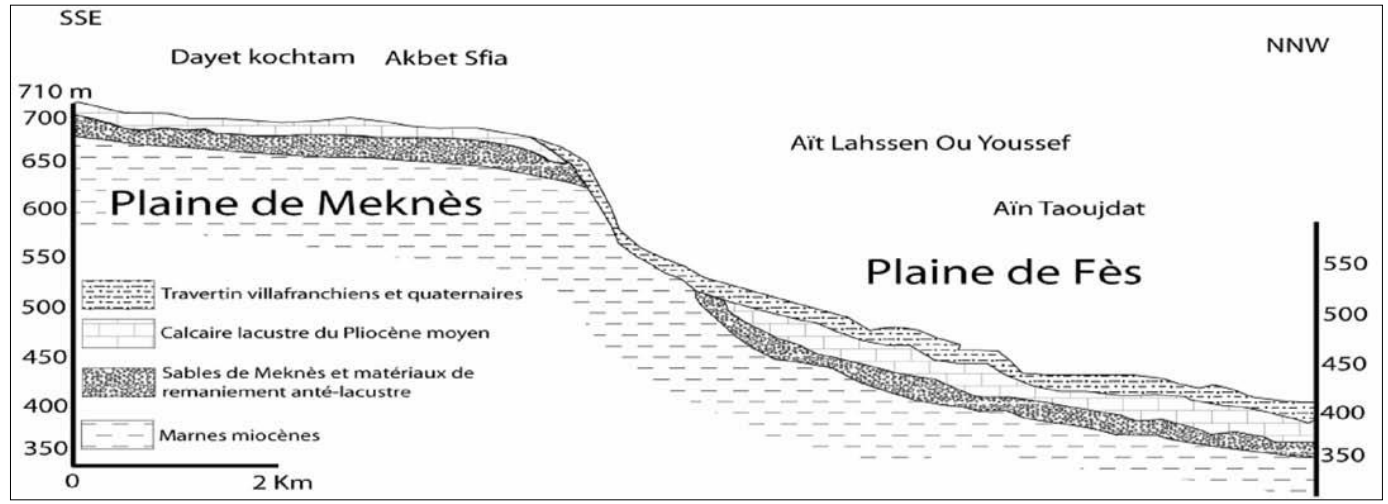

Figure 02 : Différenciation d'altitude entre la plaine de Fès et plateau de Meknès (Fassi, 1999).

\section{Lithostratigraphie}

La zone d'étude se situe dans le bassin de Saïss et particulièrement dans le plateau de Meknès, aux coordonnées Lambert X : 483600 et Y : 370000, (fig.03).

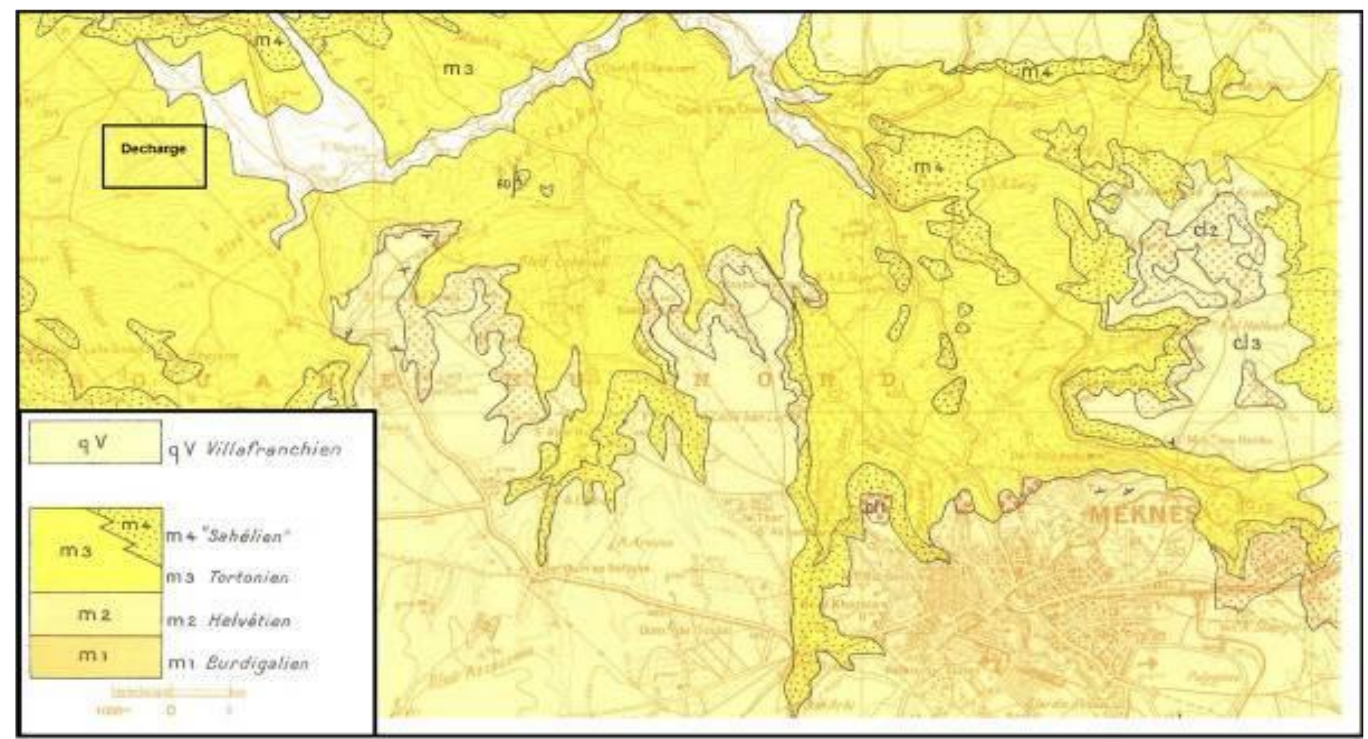

Figure 03 : Situation de la décharge sur la carte géologique de Meknès au /100 000.

Le bassin de Saïs est une large structure sédimentaire tertiaire à remplissage miocène et plio-quaternaire, mais la lithostratigraphie débute dès le Paléozoïque (fig.04). Les variations à noter sont celles des formations Plioquaternaires dans le bassin qui varient selon le contexte sédimentaire local (Harmouzi, 2010). 


\section{Paléozoïque}

Le Paléozoïque est représenté par une alternance de pélites et de bancs gréseux. Il affleure au massif hercynien central où il est en contact large avec le bassin au Sud. Le socle paléozoïque affleure au sud-ouest de la ville de Meknès au niveau d'Oued El Kell à la région d'Agouray, au niveau d'El Bhalil et au niveau d'El Hajeb.

\section{Trias}

Il est constitué essentiellement par des argiles gypsifères et salifères (dépôts évaporitiques) a intercalations de basaltes dolomitiques (fig.04). Un niveau sédimentaire carbonaté, de faible épaisseur (5m au maximum), est en permanence associé à des basaltes (Ourghache, 1987).

\section{Lias}

Le Lias du Saïss n'est connu qu'à travers les sondages réalisés dans le bassin, à l'exception de sa bordure sud-est où il arrive à l'affleurement à Bou Oughioul et montre des faciès proches de ceux des Causses (Amraoui, 2005). Les formations liasiques constituent le matériel essentiel des reliefs avec des calcaires dolomitiques inférieurs massifs et ruiniformes du Lias inférieur (Lotharingien), surmontées par des calcaires à céphalopodes et calcaires à silex du Lias moyen constitué par les étages Carixien et Domérien. Elles présentent une puissance très variable (Essahlaoui, 2000). L'épaisseur de ces formations retrouvées dans certains forages peut atteindre 280m (région de Sebâa Ayoun). Les profondeurs de ces formations sont de plus en plus grandes en allant du sud vers le nord.

\section{Miocène supérieur}

La colonne stratigraphique (fig.04), montre que la série miocène est généralement marneuse. Le passage du Lias au Miocène est matérialisé par un conglomérat de base, puis un calcaire gréseux a passées marneuses, et le reste dominé par les marnes grises du Tortonien. La limite entre le Tortonien et le Missinien est marquée par une strate de grès, surmontée par les marnes grises gréseuses (Aït Brahim, 1991 \& Essahlaoui, 2000).

\section{Pliocène}

Le Pliocène inférieur est représenté par un dépôt de sables à matrice carbonatée avec une épaisseur de 50-80m dans la région de Meknès (Boumir, 1987). Il est surmonté par des sables fauves du Pliocène moyen, d'une trentaine de mètre, déposé sur le plateau de Meknès (fig.04). Ces sables sont de couleur variable (rouge, jaune, brun, ocre, gris...etc) et constituent les futurs sables fauves (Taltasse, 1953). Le Pliocène supérieur, représenté par une alternance 
des calcaires argileux et de calcaires micritiques, est connu sous le nom de calcaires lacustres (Taltasse, 1953).

\section{Quaternaire}

La colonne se termine par un Quaternaire très diversifié (fig.04), constitué d'un complexe fluviatile (Ahmamou, 1987). Ce complexe est constitué par des tufs, des conglomérats, des lentilles de calcaires et des limons gris.

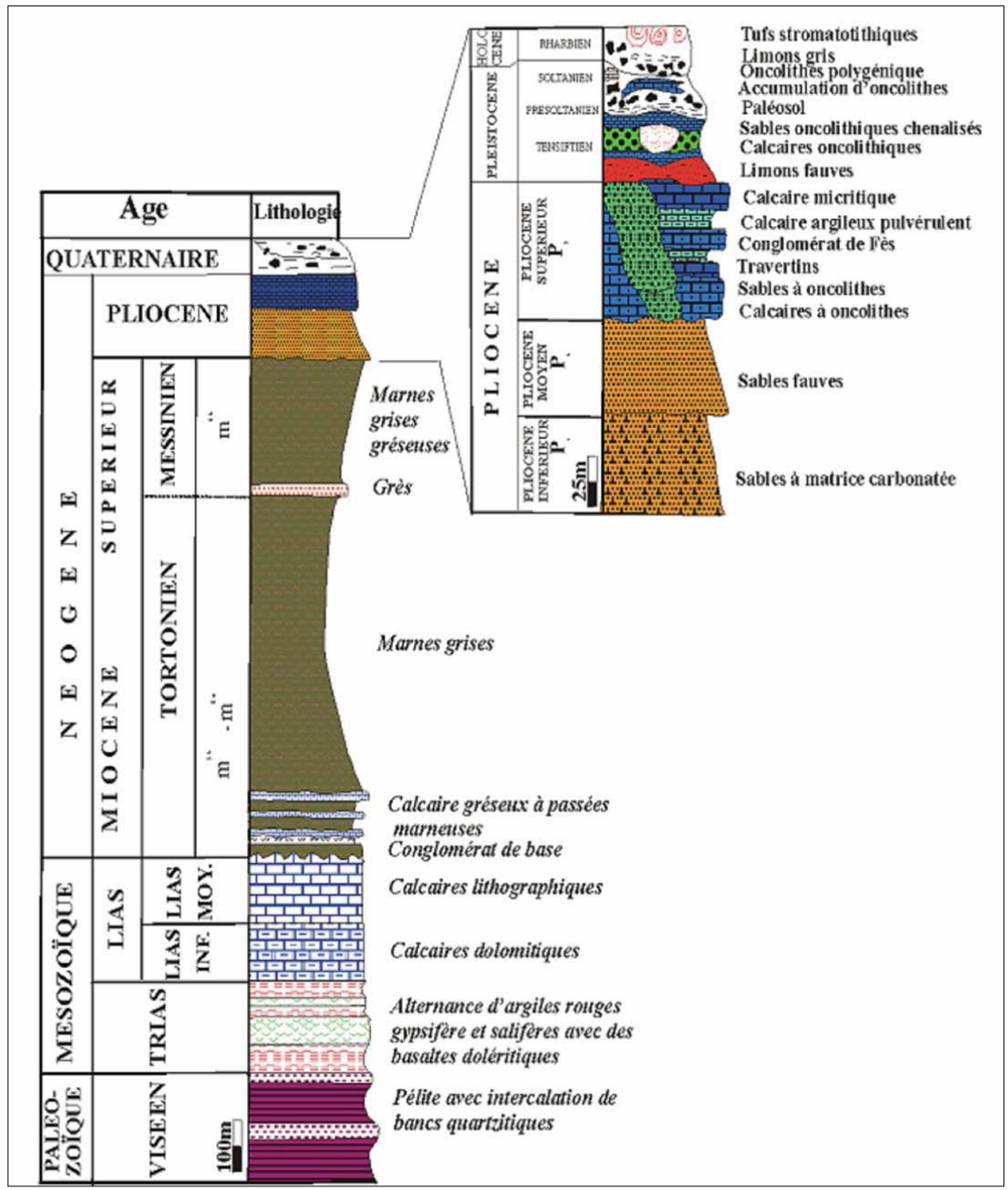

Figure 04 : Colonne stratigraphique synthétique de bassin de Saïss (Ait Brahim, 1991).

\section{Contexte hydrologique hydrogéologique}

Le réseau hydrographique au niveau du plateau de Meknès (fig.05), présente une direction qui coïncide avec la direction de la surface structurale sus-sus-est et nord-nord-ouest. La zone d'étude (décharge de Meknès) se situe 
dans le bassin versant du Rdom comprenant les oueds : Boufekrane, Ouisslane et Chajara. Le plus important de ces oueds est Boufekrane, aussi bien en raison de son débit (310 1/s en étiage) que de son déploiement au cœur de la ville (Abdouh et al., 2004). La majorité du site de décharge est drainée par l'Oued Boufekrane. On constate la présence, à l'intérieur de la décharge, de 2 thalwegs qui rejoignent l'Oued Boufekrane. Le thalweg Sud, le plus important, est partiellement rempli par les déchets. Du point de vue hydrogéologique, on distingue dans le bassin de Meknès -Fès deux nappes importantes. Il s'agit d'une part de la nappe phréatique d'age plio-quaternaire et de la nappe profonde du Lias (Tabyaoui, 2005). Comme le montre la figure 05, le site de la décharge est situé sur une zone imperméable de la nappe phréatique principale.

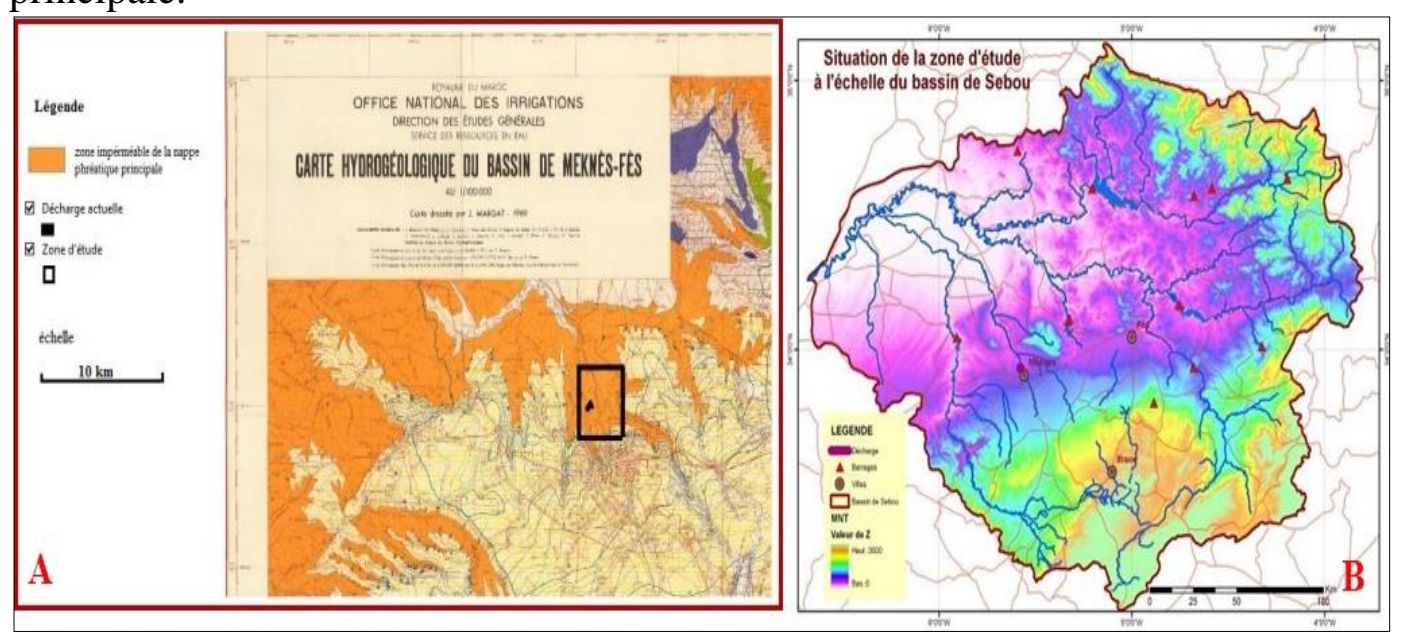

Figure 05 : le contexte et hydrogéologique (A) (Margat, 1960) et hydrologique (B) du bassin du Saïss.

\section{Matériel et méthodes}

\section{Choix des stations de prélèvement}

Au total, cinq stations ont été choisies : Quartes stations (S1, S2, S3, S4) qui correspondent à des prélèvements effectués dans l'Oued Boufekrane en aval et en amont de la décharge, et une station P5 qui correspond à des prélèvements sur un effluent de lixiviat provenant de la décharge. (fig.06). 


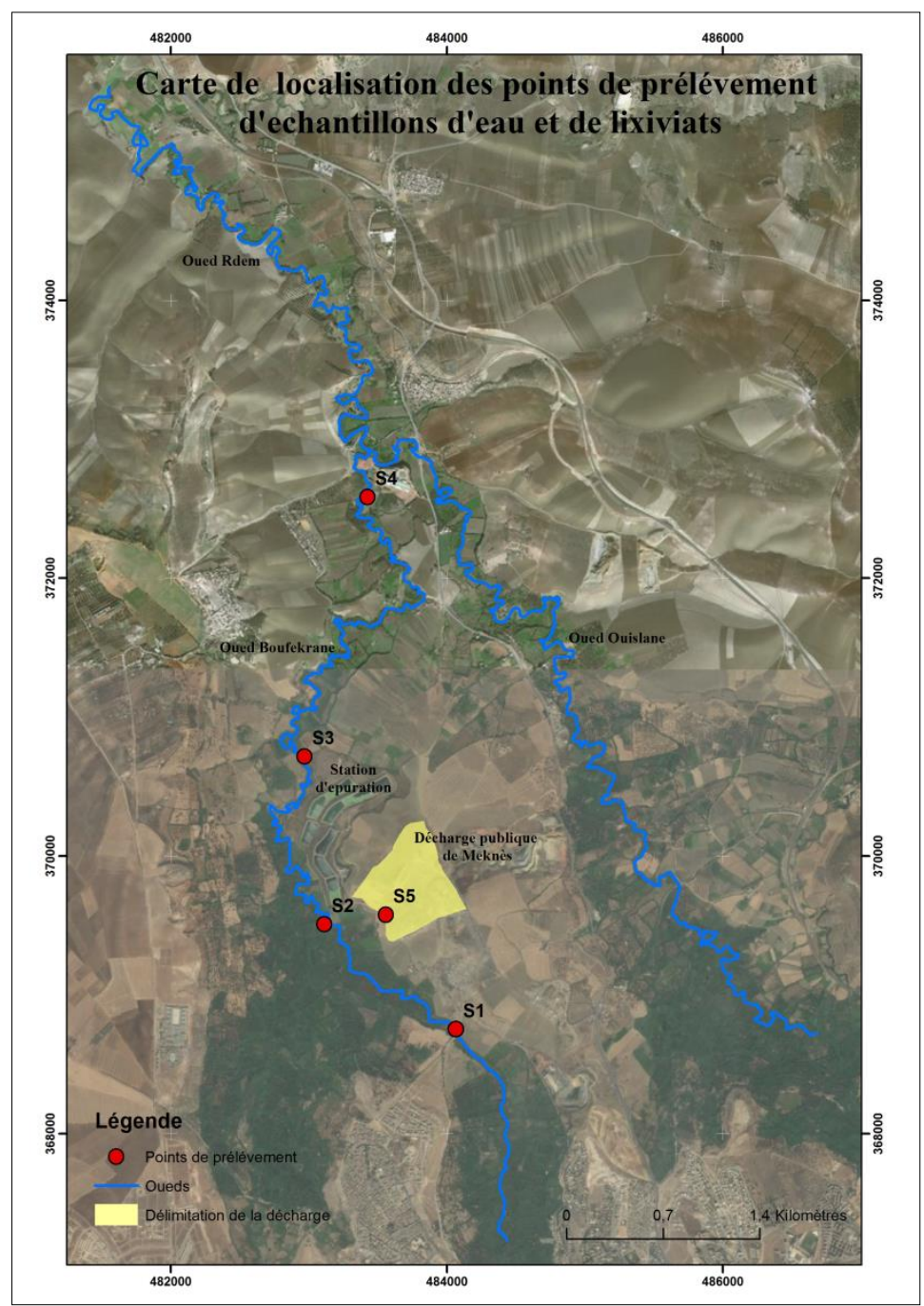

Figure 06 : Carte Situation des stations de prélèvements.

\section{1. Échantillonnage et analyses}

\section{Prélèvements}

La caractérisation des lixiviats a été réalisée sur des prélèvements effectués durant une période allant de juillet 2014 à décembre 2015 dans différents effluents des lixiviats provenant de la décharge. Pour l'échantillonnage des eaux, quatre compagnes ont été réalisées: Deux en période de hautes eaux (Décembre 2014, Novembre 2015) et deux en périodes de basses eaux (Juillet 2014, Mai 2015). 


\section{Analyses physico-chimiques}

Pour les analyses physico-chimiques, nous avons adopté les techniques décrites par Rodier et al., (2009). Au total, 8 paramètres sont étudiés. Nous distinguons les mesures in-situ et les analyses au laboratoire.

\section{Mesures effectuées sur le terrain}

Certains paramètres physico-chimiques ont été mesurés sur le terrain au moment des prélèvements. C'est le cas de la température de l'eau, du potentiel hydrogène $(\mathrm{pH})$ et de la conductivité (tab.01).

Tableau 01 : Méthodes utilisées pour les mesures in situ.

\begin{tabular}{|c|c|c|}
\hline Paramètre & Méthode de mesure & Unité \\
\hline $\mathbf{T}^{\circ} \mathrm{C}$ l'eau & $\begin{array}{l}\text { Thermomètre à mercure graduée au } \\
1 / 10 \text { de degré Celsius }\end{array}$ & $\mathbf{o C}$ \\
\hline PH & PH-mètre & $1-14$ \\
\hline Conductivité & Conductimètre & $\mu \mathrm{S} / \mathrm{cm}(25 \mathrm{oC})$ \\
\hline
\end{tabular}

\section{Analyses effectuées au laboratoire}

Les analyses des autres paramètres chimiques ont été effectuées au laboratoire. Les paramètres analysés sont: Nitrates, Oxygène dissous, Demande biologique en oxygène pendant cinq jours (DBO5), Demande chimique en oxygène (DCO) et Matières en suspension (MES). La méthodologie suivie pour analyser les différents paramètres de la qualité des eaux est résumée dans le tableau 02 .

Tableau 02 : Méthodes utilisées pour l'analyse des différents paramètres physico-chimiques.

\begin{tabular}{|l|c|c|}
\hline Paramètre & Méthode de mesure & Unité \\
\hline Nitrates & Salicylate de sodium & $\mathrm{mg} / \mathrm{L}$ \\
Oxygène dissous & $\begin{array}{c}\text { Méthode de Winkler modifiée par Alsterberg (Rodier } \\
\text { et al., 2009) }\end{array}$ & $\mathrm{mg} / \mathrm{L}$ \\
\hline DBO5 & Méthode de Winkler modifiée par Alsterberg & $\mathrm{mg} / \mathrm{L}$ \\
\hline DCO & Dichromate de potassium & $\mathrm{mg} / \mathrm{L}$ \\
\hline MES & Filtration sur membrane & $\mathrm{mg} / \mathrm{L}$ \\
\hline
\end{tabular}

Résultats et discussions

Analyses physico-chimiques des lixiviats

Les résultats de l'analyse physico-chimique des lixiviats issus de la décharge publique de Meknès sont présentés dans le tableau suivant : 
Tableau 03 : Caractéristiques physico-chimique des lixiviats de la décharge de la ville de Meknès

\begin{tabular}{|l|c|c|c|c|c|}
\hline & Juillet 2014 & décembre 2014 & Mai 2015 & novembre-2015 & moyenne \\
\hline Température & 22,3 & 17 & 21 & 21 & 20,33 \\
\hline Ph & 8,25 & 7,35 & 7,80 & 6,90 & 7,48 \\
\hline Conductivité & 28593 & 36000 & 24000 & 34628 & 30805 \\
\hline DBO5 & 725 & 290 & 580 & 796 & 598 \\
\hline DCO & 3690 & 1812 & 3120 & 4765 & 3346 \\
\hline DBO5/DCO & 0,20 & 0,16 & 0,19 & 0,17 & 0,18 \\
\hline MES & 1500 & 285 & 1050 & 495 & 832,50 \\
\hline Chlorures & 8140 & 2400 & 4400 & 5600 & 5144 \\
\hline Sulfates & 920 & 276 & 450 & 510 & 539 \\
\hline Nitrates & 10 & 7,3 & 11,2 & 6,35 & 8,71 \\
\hline
\end{tabular}

La couleur brunâtre et l'odeur fécaloïde des lixiviats de la décharge publique de Meknès nous donnent une idée sur le degré de pollution avancée la dégradation de la qualité des eaux. Le $\mathrm{pH}$ des lixiviats de la décharge publique de Meknès est légèrement neutre à alcalin avec des valeurs qui varient entre 6,9 et 8,2 avec une moyenne annuelle de 7,48. Il augmente en fonction du temps du séjour des lixiviats qui s'appauvrit en composés organiques volatils au fur et a mesure du vieillissement de la décharge (Kjeldsen et al., 2002).Ces valeurs confirment celles enregistrées par (Mejraoua et al., 2017) qui varient entre 6,42 et 8,99 , sont proches de celles soulevées à Larache(7,87),(Er-raioui et al., 2011) et largement supérieures à celles enregistrées à Agadir(4,3),(Jirou et al., 2014).

Les valeurs de la conductivité électrique enregistrées au cours de cette étude sont élevées et varient entre 24000 et $36000 \mu \mathrm{s} / \mathrm{cm}$ avec une moyenne annuelle de $30805 \mu \mathrm{s} / \mathrm{cm}$. Ces valeurs sont largement supérieures à la valeur limite de rejets directs dans le milieu récepteur qui est estimée à $2700 \mu \mathrm{s} / \mathrm{cm}$ (Ministère délègue auprès du Ministre de l'Énergie, des Mines, de l'eau et de l'environnement charge de l'eau du Maroc, 2014), ce qui explique que les lixiviats de la décharge représentent une salinité très importante. Ce degré important de salinité provient d'après (Hakkou et al., 2001) de la minéralisation de la matière organique et du lessivage des sels solubles dans les déchets.

La valeur moyenne annuelle de la conductivité électrique à la décharge de Meknès estimée à $30805 \mu \mathrm{s} / \mathrm{cm}$ est supérieure à celle enregistrée à Kenitra (22792 $\mu \mathrm{S} / \mathrm{CM}$ ), (El Markhi et al, 2013). Par contre elle est largement inférieure à celle enregistrée à Larache $(64600 \mu \mathrm{s} / \mathrm{cm})$, (Er-raioui et al, 2011) et à celle d'Essaouira $(39983 \mu \mathrm{s} / \mathrm{cm})$ (Chiguer et al., 2016).

Les concentrations en matière en suspension enregistrent des valeurs comprises entre 285 et $1500 \mathrm{mg} / \mathrm{l}$ avec une moyenne annuelle de $832 \mathrm{mg} / \mathrm{l}$. Ces 
valeurs sont supérieures à celles enregistrées à Essaouira $(146 \mathrm{mg} / \mathrm{l}),($ Chiguer et al., 2016) et à El Mohammedia (660,5 mg/1),(Chiguer et al., 2016) mais qui reste largement inférieures à celles enregistrées à la décharge d'Agadir (12560mg/l),), (Jirou et al., 2014). Ces concentrations importantes, représentent un indicateur de la forte charge organique et minérale dues à la nature des déchets.

Les teneurs en nitrates sont comprises entre 6,35 et $11 \mathrm{mg} / \mathrm{l}$ avec une moyenne annuelle de $8,71 \mathrm{mg} / \mathrm{l}$ qui reste largement inférieure à la valeur limite de rejets directs dans le milieu récepteur qui est estimée à $50 \mathrm{mg} / \mathrm{l}$ (Ministère délègue auprès du Ministre de l'Énergie, des Mines, de l'eau et de l'environnement charge de l'eau du Maroc, 2014). Les faibles concentrations des forme oxydées de l'azote (Nitrates et Nitrites) est le résultat de l'absence d'oxygène dissous dans les lixiviats (Hakkou et al., 2001), ce qui nous amène à déduire que le phénomène d'anaérobiose est prédominant dans la décharge.

Les valeurs de la DBO5 sont très élevées, elles varient entre 290 et 796 $\mathrm{mg} / \mathrm{l}$, avec une moyenne annuelle de $598 \mathrm{mg} / \mathrm{let}$ sont largement supérieures à la valeur limite de rejets directs qui est estimée à $100 \mathrm{mg} / \mathrm{l}$. Ces résultats sont très proches de celles observés à la décharge d'Essaouira $(632 \mathrm{mg} / \mathrm{l})$, (Chiguer et al., 2016) est largement inférieurs à celles des décharges de Fès (1700mg/l),(Bouaouine et al., 2015) et d'Agadir (44000mg/l),(Jirou et al., 2014).

De même pour la DCO dont les teneurs importantes variant entre 1812 et4765 mg/l avec une moyenne annuelle de 3346mg/l. Ces valeurs dépassant ainsi la valeur limite de rejets directs qui est estimée à $500 \mathrm{mgO}_{2} / 1$.Ces valeurs sont largement inférieurs à celles des décharges de Fès $(5400 \mathrm{mg} / \mathrm{l})$, ( Bouaouine et al., 2015) et d'Agadir (72000mg/l)),(Jirou et al., 2014) ,d'où l'importance de la pollution organique des lixiviats de la décharge publique de Meknès et qui est fortement liée à la dégradation incomplète de la matière organique des déchets.

Le rapport DBO5/DCO qui renseigne sur l'étape de fermentation de la décharge, varie entre 0,16 et 0,2.Selon (Kouassi et al., 2007), ce rapport est supérieur à 0,5 pour les lixiviats jeunes, inferieur à 0,1 pour les lixiviats stabilisés et compris entre 0,1 et 0,5 pour les lixiviats intermédiaires. Cela explique qu'on est en présence de lixiviats intermédiaires dont la matière organique n'a pas atteint le stade final de la dégradation et qu'à ce stade, la décharge traverse la phase instable de la fermentation méthanique (Hakkou et al,. 2001).

\section{Analyse descriptive des paramètres physico-chimiques de la qualité de l'eau de l'Oued Boufekrane}

Les résultats des analyses des paramètres physico-chimiques effectuées sur les eaux de surface et précisément sur les quatre stations situées sur l'Oued 
Boufekrane, une à l'amont et trois autres à l'avale de la décharge publique de la ville de Meknès sont présentés comme suit (fig.07) :

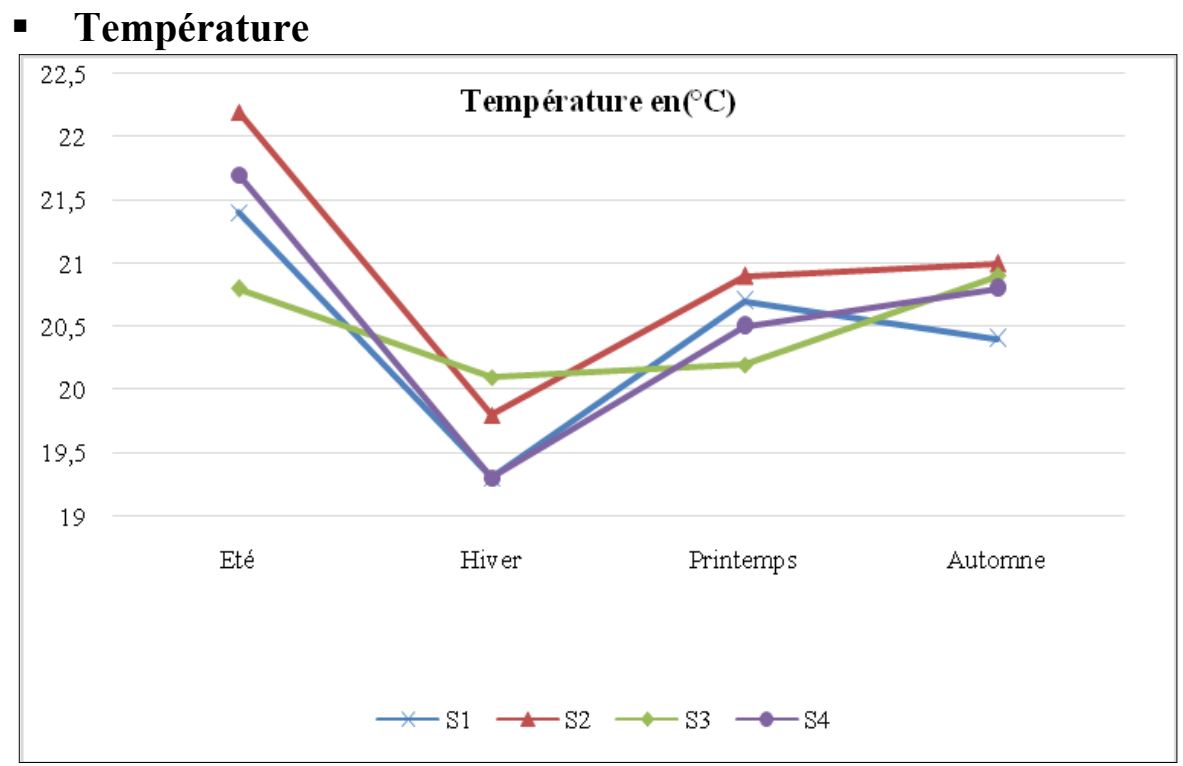

Figure 07 : Évolution spatio-temporelle et valeurs de la température de l'eau

La variabilité spatio-temporelle des températures des eaux de l'oued Boufekrane, enregistre une valeur $21,4^{\circ} \mathrm{C}$ en amont de la décharge publique de Meknès (station S1). La température est augmentée à l'aval de cette dernière (station S2) à $22,2^{\circ} \mathrm{C}$ et ce juste après la confluence de l'Oued avec l'effluent des lixiviat émanant de la décharge, puis diminue juste après les déversements de la station d'épuration dans l'Oued (station S3) à $20,8^{\circ} \mathrm{C}$. Elle connait une légère augmentation encore une fois pour atteindre un pic maximal d'ordre de $21,7^{\circ} \mathrm{C}$ vers l'aval juste avant la confluence de $1^{\prime}$ 'Oued Boufekrane et Oued Ouislane (station S4). Les variations saisonnières de la température des eaux de l'oued sont négligeables du fait que depuis quelques années, l'hydrologie de l'oued Boufekrane a connu un changement de ces caractéristiques physiques et chimiques à l'amont de la ville de Meknès par la création d'un lac réservoir sur ce cours d'eau. Ce plan vaste qui occupe les anciennes carrières permet une homogénéisation des apports de l'oued, ce qui donne un gradient constant des paramètres de température, de $\mathrm{pH}$ et de conductivité électrique à la station de référence de ce travail (S1). Ce gradient constant est influencé aussi par les déviations des rejets des eaux usées de la ville de Meknès à l'oued Boufekrane pour des fins d'irrigation clandestines. 


\section{- Potentiel hydrogène $(\mathrm{pH})$}

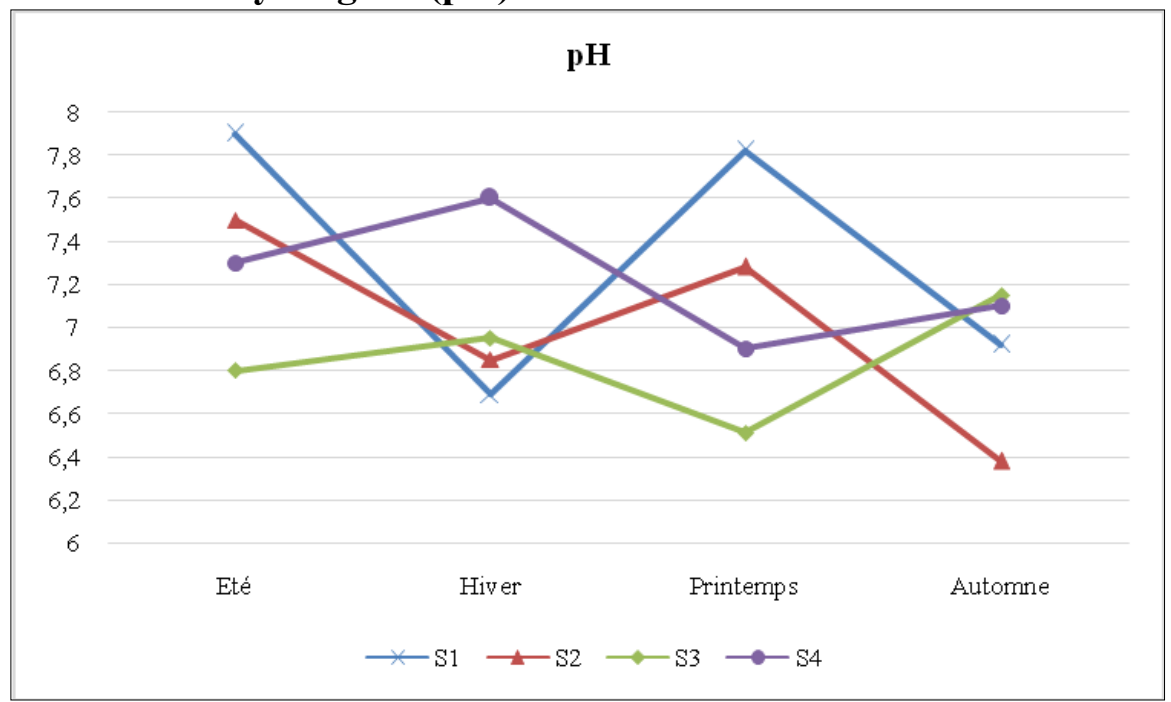

Figure 08 : Évolution spatio-temporelle et valeurs du pH de l'eau

Le suivi du paramètre $\mathrm{pH}$ sur les quatre stations a mis en évidence l'impact des rejets des margines sur l'oued Boufekrane qui reçoit les effluents de plusieurs huileries situées en amont de la station de référence S1. De ce fait, on constate une acidification du cours d'eau en période de trituration de l'huile d'olive qui commence en mois d'octobre et s'étale jusqu'à la fin du mois de février qui connait des déversements importants des bassins de stockage des margines. Les valeurs du pH varient de 6,69 à 6,92 en hiver et automne.

Ces valeurs de $\mathrm{pH}$ impactent aussi les variations observées à la station S2 qui suivent le même gradient de la station de référence.

A la station $\mathrm{S} 3$, on note une régression de la valeur du $\mathrm{pH}$ estivale et printanière en raison de l'augmentation du pouvoir épuratoire des effluents émanant de la station d'épuration de la ville qui peuvent être justifiés par la présence des bactéries acidophiles dans ces rejets.

Le $\mathrm{pH}$ à cette station connait une légère tendance à la hausse en hiver et en automne à causse de l'augmentation des apports hydrique de l'oued Boufekrane dû essentiellement aux précipitations. A la station S4, l'autoépuration a permit à l'oued d'avoir ces caractéristiques d'origines en amont de la ville de Meknès explicitées par (Larif, 2012).

Les valeurs enregistrées dans les eaux de l'Oued Boufekrane se trouvant dans la zone d'étude sont comprises entre 6,38 et 7,9 et dévoilent un léger gradient décroissant en amont de la décharge publique. Vers l'aval de la station d'épuration et la confluence de l'Oued Boufekrane et Oued Ouislane, car la décomposition de la matière organique liée à l'activité bactérienne favorise la diminution du $\mathrm{pH}$ et semble être dépassée par l'apport des rejets polluants (Ansa-Asare et al., 1999 ; Neal et al., 2000a). 
- Conductivité

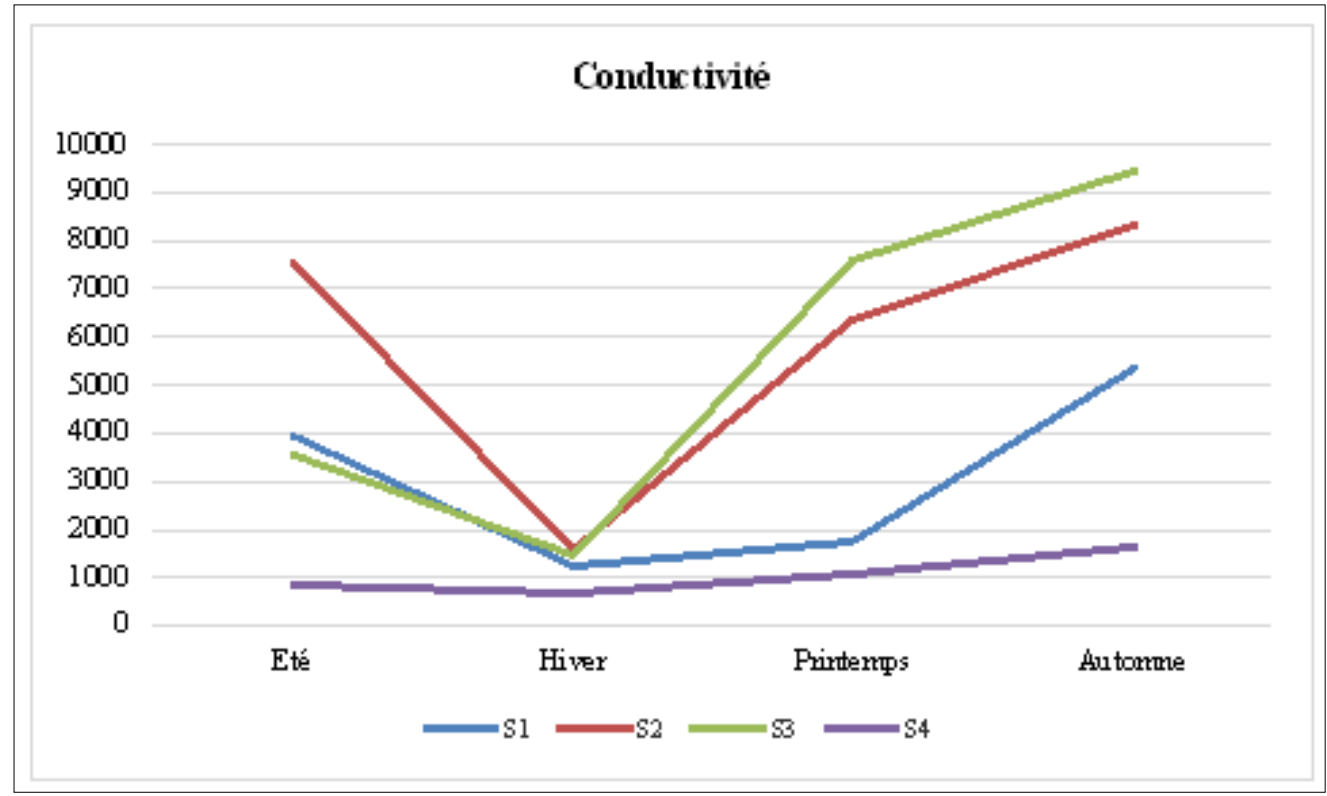

Figure 09 : Évolution spatio-temporelle et valeurs de la conductivité électrique

La conductivité des eaux de l'oued évolue selon un gradient croissant de la station $\mathrm{S} 1$ vers $\mathrm{S} 2$, puis elle diminue légèrement vers la station $\mathrm{S} 3$ et continue sa régression importante vers la station S4. L'Oued Boufekrane passe ainsi d'une conductivité moyenne annuelle de $3058 \mu \mathrm{S} / \mathrm{cm}$ en S1 à $5939 \mu \mathrm{S} / \mathrm{cm}$ en $\mathrm{S} 2$, diminue à $5562 \mu \mathrm{s} / \mathrm{cm}$ en S3 et chute à $1054 \mu \mathrm{s} / \mathrm{cm}$ à la station S4.

La conductivité variante entre 694 et $9642 \mu \mathrm{s} / \mathrm{cm}$ nous informe sur le degré de minéralisation important de l'amont vers l'aval qui trouve son origine dans les influences d'origines anthropiques et qui proviennent certainement des rejets industriels des huileries (en amont de la station de référence S1) en automne. Durant la saison d'été, on constate une élévation de ce paramètre dû certainement à la forte évaporation et au bas débit de l'eau de l'oued Boufekrane en faveur de la prédominance des rejets des lixiviats (en aval de la station de référence $\mathrm{S} 1$ ) et des effluents émanant de la station d'épuration(S3) qui assure seulement un traitement primaire des eaux usées de la ville.

En hiver la diminution de la conductivité pourrait être expliquée par le phénomène de dilution que connaît l'oued suite aux précipitations en période hivernale 
- Demande Chimique en Oxygène

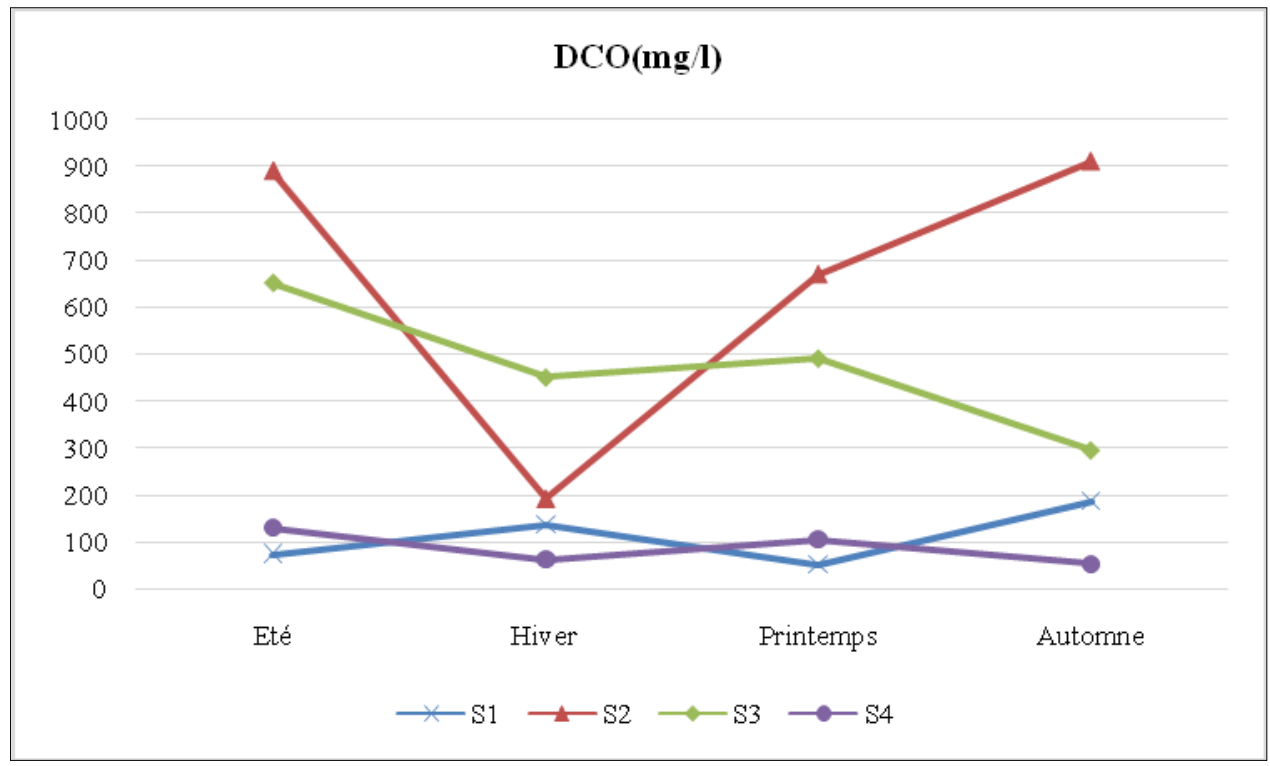

Figure 10 : Évolution spatio-temporelle et valeurs de la DCO

L'analyse des résultats présentés dans le tableau 7 montre que La DCO des eaux de l'oued connait une augmentation importante de la station S1 vers S2 puis elle diminue selon un gradient décroissant vers les stations S3 et S4. L'Oued Boufekrane passe ainsi d'une moyenne annuelle de la DCO estimée à $112 \mathrm{mg} / 1$ en $\mathrm{S} 1$ à $666 \mathrm{mg} / 1$ en $\mathrm{S} 2$, diminue légèrement à $472 \mathrm{mg} / 1$ en S3 et continue sa régression pour atteindre $88 \mathrm{mg} / \mathrm{l}$ à la station $\mathrm{S} 4$.

L'évolution saisonnière de la DCO (tableau 7) montre que les teneurs les plus élevées sont enregistrées en automne $(910 \mathrm{mg} / \mathrm{l}$ en S2), en été (890 $\mathrm{mg} / \mathrm{l}$ en $\mathrm{S} 2$ et $650 \mathrm{mg} / \mathrm{l}$ en S3) et en Printemps $(670 \mathrm{mg} / \mathrm{l}$ en S2) dépassant ainsi la valeur limite de rejets directs qui est estimée à $500 \mathrm{mg} / \mathrm{l}$. les valeurs de la DCO connaissent une tendance à la baisse en hiver à causse de l'augmentation des apports hydrique de l'oued Boufekrane dû essentiellement aux précipitations.

L'augmentation dans la station S2 et S3 de la demande chimique en oxygène pourrait être liée au degré croissant de la pollution en amont. Ainsi, l'évolution saisonnière de ce paramètre pourrait s'expliquer en automne, par les rejets importants et inquiétants des margines, et durant toute l'année par les rejets excessifs des lixiviats issus de la décharge publique et des effluents émanant de la station d'épuration de la ville. 
- Demande Biologique en Oxygène en 5 jours

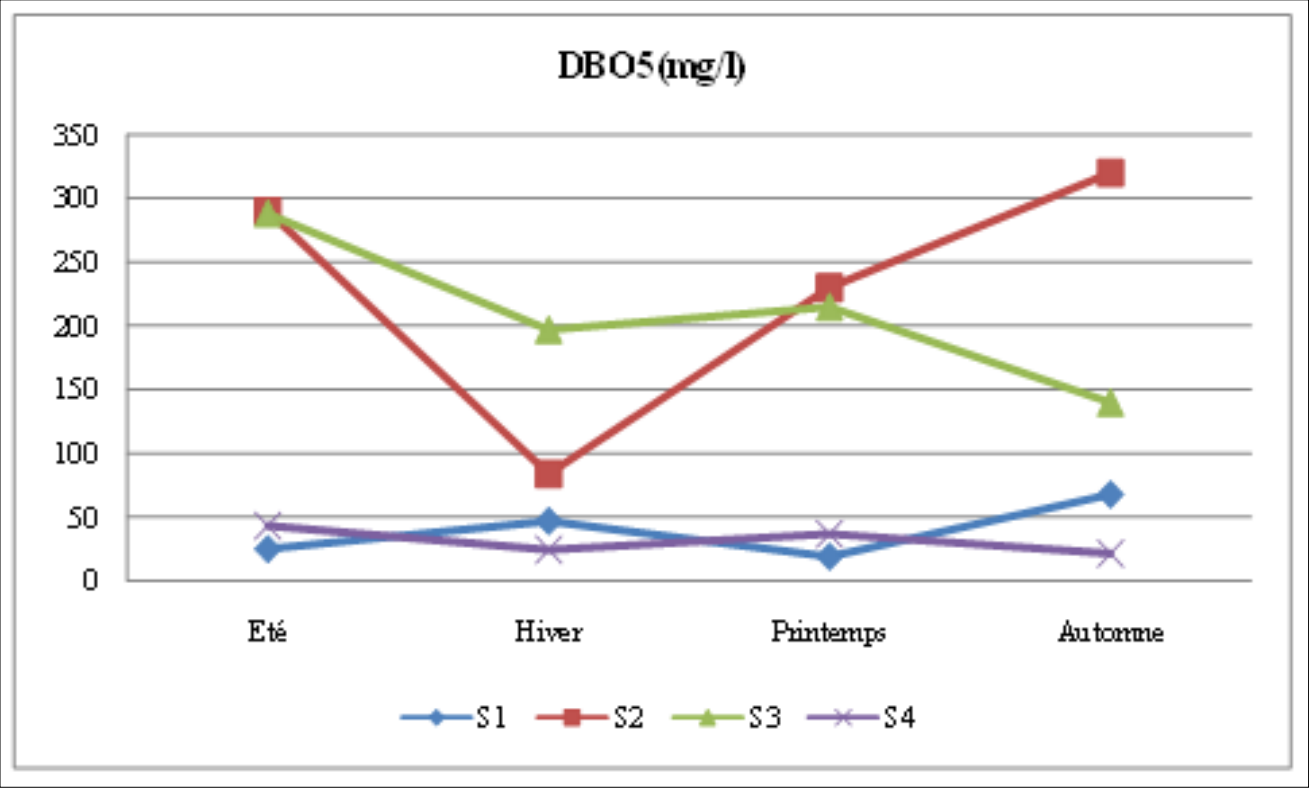

Figure 11 : Évolution spatio-temporelle et valeurs de la DBO5

L'analyse des résultats présentés dans le tableau 8 montres que La DBO5 des eaux de l'oued connait une augmentation importante de la station $\mathrm{S} 1$ vers $\mathrm{S} 2$, puis elle diminue légèrement vers la station $\mathrm{S} 3$ et continue sa régression importante vers la station S4. L'Oued Boufekrane passe ainsi d'une moyenne annuelle de la DBO5 estimée à $40 \mathrm{mg} / 1$ en $\mathrm{S} 1$ à $231 \mathrm{mg} / 1$ en $\mathrm{S} 2$, diminue légèrement à $210 \mathrm{mg} / \mathrm{l}$ en $\mathrm{S} 3$ et chute à $88 \mathrm{mg} / \mathrm{l}$ à la station $\mathrm{S} 4$.

L'évolution saisonnière de la DBO5 (tableau 8) montre que les teneurs les plus élevées sont enregistrées en automne ( $320 \mathrm{mg} / 1$ en S2), en été (290 $\mathrm{mg} / \mathrm{l}$ en S2 et $288 \mathrm{mg} / \mathrm{l} \mathrm{en} \mathrm{S3}$ ) et en Printemps ( $230 \mathrm{mg} / \mathrm{l}$ en S2 et $215 \mathrm{mg} / \mathrm{l} \mathrm{en}$ S3) dépassant ainsi la valeur limite de rejets directs qui est estimée à $100 \mathrm{mg} / \mathrm{l}$.

Les résultats illustrés par la figure 5, montrent une chute des valeurs de la DBO5 des stations S2, S3 et S4. Ce rabattement enregistré en hiver, est dû essentiellement au phénomène de dilution causé par les précipitations.

Comme pour la DCO, L'augmentation dans la station S2 et S3 de la demande biologique en oxygène pendant cinq jours pourrait être liée au degré croissant de la pollution en amont vers l'aval. L'évolution saisonnière de ce paramètre pourrait s'expliquer en automne et en hivers par les lessivages des sols et par les rejets importants des margines, en été par la diminution importante du débit de l'oued associée à la prédominance des lixiviats issus de la décharge publique, des effluents émanant de la station d'épuration de la ville et par l'instauration des conditions de dégradation de la matière organique par les microorganismes dont l'activité s'intensifie avec la diminution de la vitesse d'écoulement et avec le réchauffement des eaux. Au printemps, le débit assez 
important de l'oued associée à l'absence des rejets des margines, contribuent relativement à la dilution de la charge organique émanant de la décharge publique et $\mathrm{d}$ la station d'épuration de la ville de Meknès.

A la station S4, l'autoépuration a permit à l'oued d'avoir ces caractéristiques d'origines en amont de la ville de Meknès explicitées par (Larif, 2012).

\section{- Oxygène dissous}

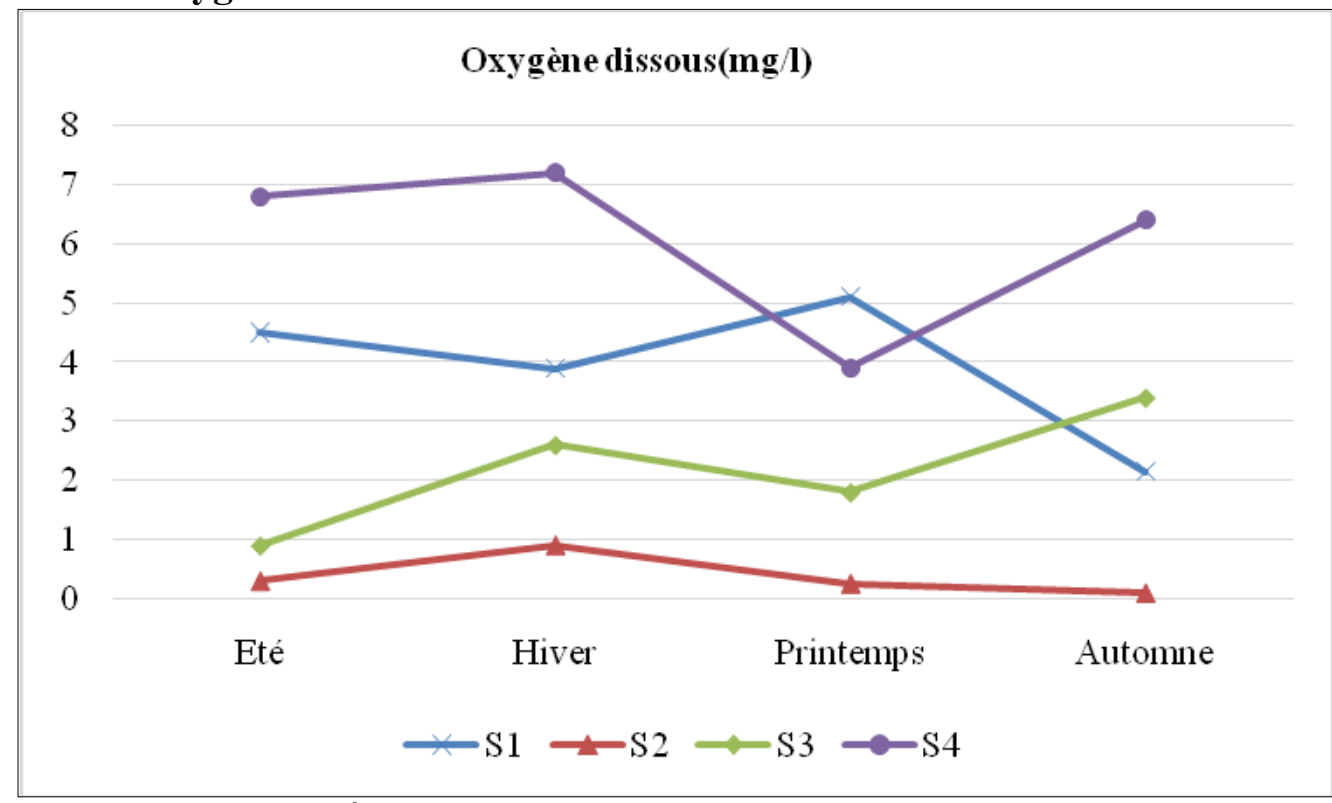

Figure 12 : Évolution spatio-temporelle et teneurs en oxygène dissous

La variabilité spatio-temporelle de l'oxygène dissous des eaux de l'oued Boufekrane (figure 6), enregistre une valeur maximale de $5,1 \mathrm{mg} / 1$ en printemps en amont de la décharge publique de Meknès (station de référence $\mathrm{S} 1$ ). Elle diminue à l'aval de cette dernière (station S2) à $0,1 \mathrm{mg} / \mathrm{l}$ en automne et ce juste après la confluence de l'Oued avec l'effluent des lixiviat émanant de la décharge, puis recommence à augmenter juste après les déversements de la station d'épuration dans l'Oued (station S3) à 2,6mg/l. En hiver elle connait une augmentation encore une fois pour atteindre un pic maximal d'ordre de 7,2 $\mathrm{mg} / \mathrm{l}$ vers l'aval juste avant la confluence de l'Oued Boufekrane et Oued Ouislane (station S4).

L'évolution saisonnière des teneurs en oxygène dissous (tableau 9) montre qu'elles sont les plus faibles en été et en automne et qu'elles augmentent en printemps et en hiver.

Les résultats obtenus en été et en automne pourraient être attribués, essentiellement, à l'effet des températures élevées des eaux, à la prédominance des eaux usées et à la diminution importante du débit de l'oued. Ces conditions sont favorables à une multiplication bactérienne importante et donc à la 
consommation d'oxygène. L'augmentation des teneurs en oxygène dissous en hiver et en printemps pourrait être attribuée à un ensemble de facteurs, entre autres, la diminution de la température de l'eau et l'augmentation du débit de l'oued et donc à un brassage important de la masse d'eau.

- Nitrates

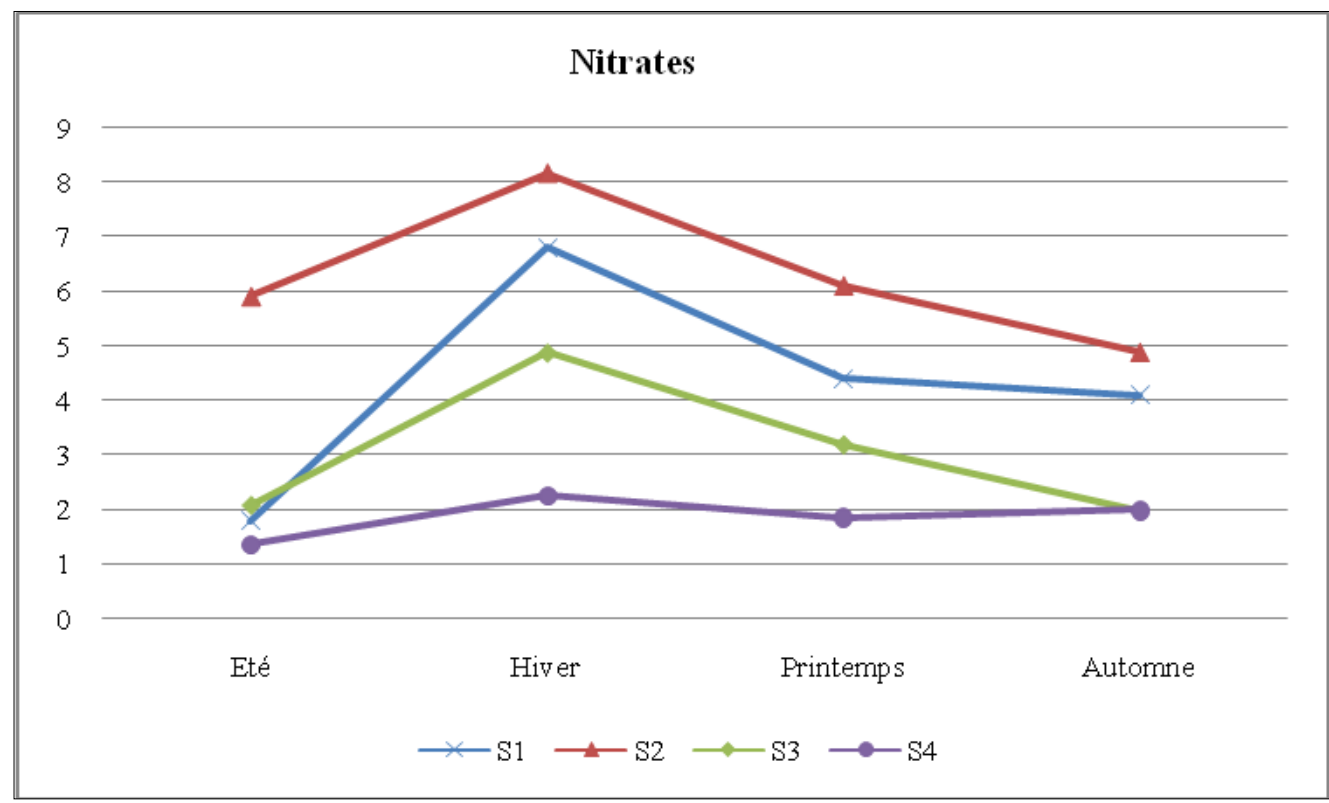

Figure 13 : Evolution spatio-temporelle et des teneurs des nitrates

Au niveau des eaux de l'oued, les concentrations minimales en nitrates ont été enregistrées en été $(1,36 \mathrm{mg} / \mathrm{l}$ à $\mathrm{S} 4$ et $1,8 \mathrm{mg} / \mathrm{l}$ à $\mathrm{S} 1)$ et en automne $(1,98$ $\mathrm{mg} / \mathrm{l}$ en S3 et S4), alors que les teneurs maximales ont été observées en hivers $(8,16 \mathrm{mg} / \mathrm{l}$ en $\mathrm{S} 2)$. Le suivi saisonnier des nitrates (tableau 10) a montré que pendant la période hivernale, les stations étudiées présentent plus de nitrates que pendant les autres saisons. Ceci peut être dû essentiellement à l'usage excessif des engrais pour l'agriculture en cette période (Dumontier et al., 1995 ; Neal et al., 2000 ; Benabdellouahad, 2006) et aux apports des eaux qui lessivent les terrains limitrophes pendant la saison pluvieuse.

Les rejets des lixiviats pourraient contribuer à cette contamination. Effectivement, on peut déduire que la concentration maximale enregistrée en hivers à la station $\mathrm{S} 2(8,16 \mathrm{mg} / \mathrm{l})$ est liée, et à l'accentuation du phénomène du lessivage des sols et au déversement sans aucun traitement préalable des margines dans l'oued enregistré à la station de référence $\mathrm{S} 1(6,8 \mathrm{mg} / 1)$, et aux apports des effluents des lixiviats issus de la décharge. La période estivoautomnale est caractérisée par la prédominance des lixiviats, des apports de la station d'épuration et par les températures relativement élevées de l'eau. La diminution des teneurs en été $(1,8 \mathrm{mg} / \mathrm{l})$ et en automne $(1,98 \mathrm{mg} / \mathrm{l})$ pourrait 
s'expliquer par la diminution de la pollution diffuse ; alors que la période printanière est caractérisée par le début de diminution du débit de l'oued et par le retour progressif, à partir de la fin de la saison, aux conditions de la période sèche. Ces fluctuations temporelles sont en accord avec celles rapportées par (Aboulkacem, 2007 ; El Addouli, 2010 ; Karrouch, 2010).

\section{- Chlorures}

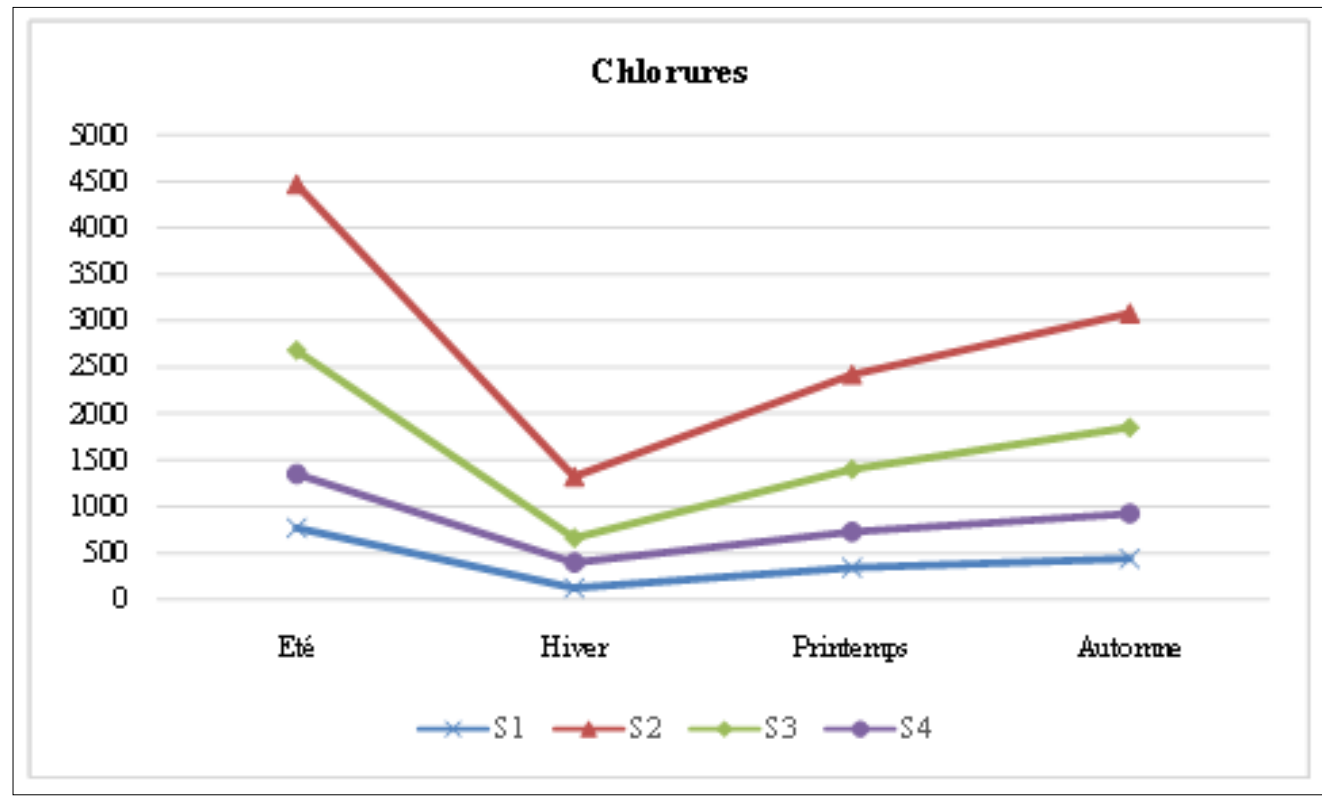

Figure14 : Evolution spatio-temporelle et valeurs des chlorures.

L'analyse des résultats présentés dans le tableau 11 montres que les teneurs en chlorures des eaux de l'oued connaissent une augmentation importante de la station $\mathrm{S} 1$ vers $\mathrm{S} 2$, puis elles diminuent légèrement vers la station S3 et continuent leur régression importante vers la station S4. L'Oued Boufekrane passe ainsi d'une moyenne annuelle des chlorures estimée à 418 $\mathrm{mg} / 1$ en $\mathrm{S} 1$ à $2222 \mathrm{mg} / 1$ en $\mathrm{S} 2$, diminue légèrement à $1647 \mathrm{mg} / 1$ en $\mathrm{S} 3$ et chute à $849 \mathrm{mg} / \mathrm{l}$ à la station $\mathrm{S} 4$.

L'évolution saisonnière des concentrations en chlorures (tableau 11) montre que celles les plus élevées sont enregistrées à la station S2 $(4470 \mathrm{mg} / 1$ en été, $1850 \mathrm{mg} / \mathrm{l}$ en automne, $1400 \mathrm{mg} / \mathrm{l}$ en Printemps et $640 \mathrm{mg} / \mathrm{l}$ en hivers) suivi de la station S3 (2680 mg/l en été, $3080 \mathrm{mg} / 1$ en automne, $2420 \mathrm{mg} / 1 \mathrm{en}$ Printemps et $1320 \mathrm{mg} / \mathrm{l}$ en hivers) dépassant ainsi la valeur limite de rejets directs qui est estimée à $750 \mathrm{mg} /$. Ces forts teneurs peuvent être expliqués par les déversements des lixiviats de la décharge et des eaux usées partiellement traitées de la station d'épuration de la ville (traitement primaire). Les hautes valeurs enregistrées à la station de référence S1 en automne sont dues essentiellement aux rejets directs des margines par les égouts de l'huilerie 
dont la forte salinité est due à l'ajout du sel pendant la conservation. En hiver, on observe une chute des concentrations des chlorures dans les stations S1, S2, S3 et S4. Cet abattement, est dû essentiellement au phénomène de dilution causé par les précipitations. L'allure générale de cette évolution rappelle celle de la conductivité.

\section{- Sulfates}

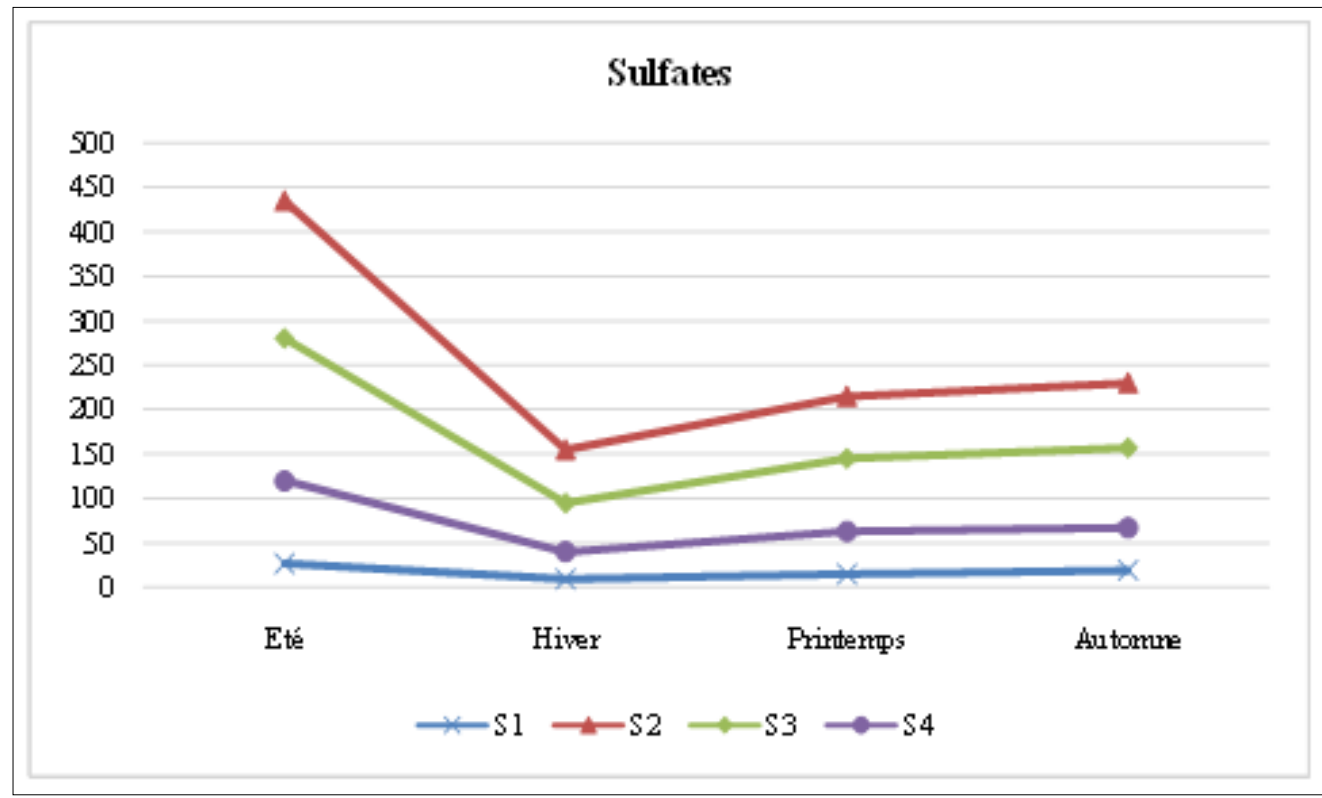

Figure 15 : Évolution spatio-temporelle et valeurs des chlorures

Comme les chlorures, le tableau 12 montres que les teneurs en sulfates des eaux de l'oued augmentent de la station S1 vers S2, puis elles diminuent vers l'aval (S3 et $\mathrm{S} 4$ ), passant d'une moyenne annuelle estimée à $17,65 \mathrm{mg} / 1$ en $\mathrm{S} 1$ à $259 \mathrm{mg} / 1$ en $\mathrm{S} 2$, diminue légèrement à $169 \mathrm{mg} / 1 \mathrm{en} \mathrm{S3}$ et chute à 72 $\mathrm{mg} / \mathrm{l}$ à la station $\mathrm{S} 4$.

L'allure générale de cette évolution nous rappelle celle de la conductivité et des chlorures. En effet, l'évolution saisonnière des concentrations en sulfates (tableau 11) montre que celles les plus élevées sont enregistrées en été à la station S2 (435 mg/l en été,) suivi de la station S3 (280 $\mathrm{mg} / \mathrm{l}$ ) dépassant ainsi la valeur limite de rejets directs qui est estimée à 200 $\mathrm{mg} /$. Ce dépassement est enregistré seulement dans les stations S2 et S3 du essentiellement à la pollution par les effluents des lixiviats et les apports de la station d'épuration. On note une diminution des teneurs en sulfates en hivers, et ce à cause du phénomène de dilution causé par la pluviométrie. 
- Solides en suspension

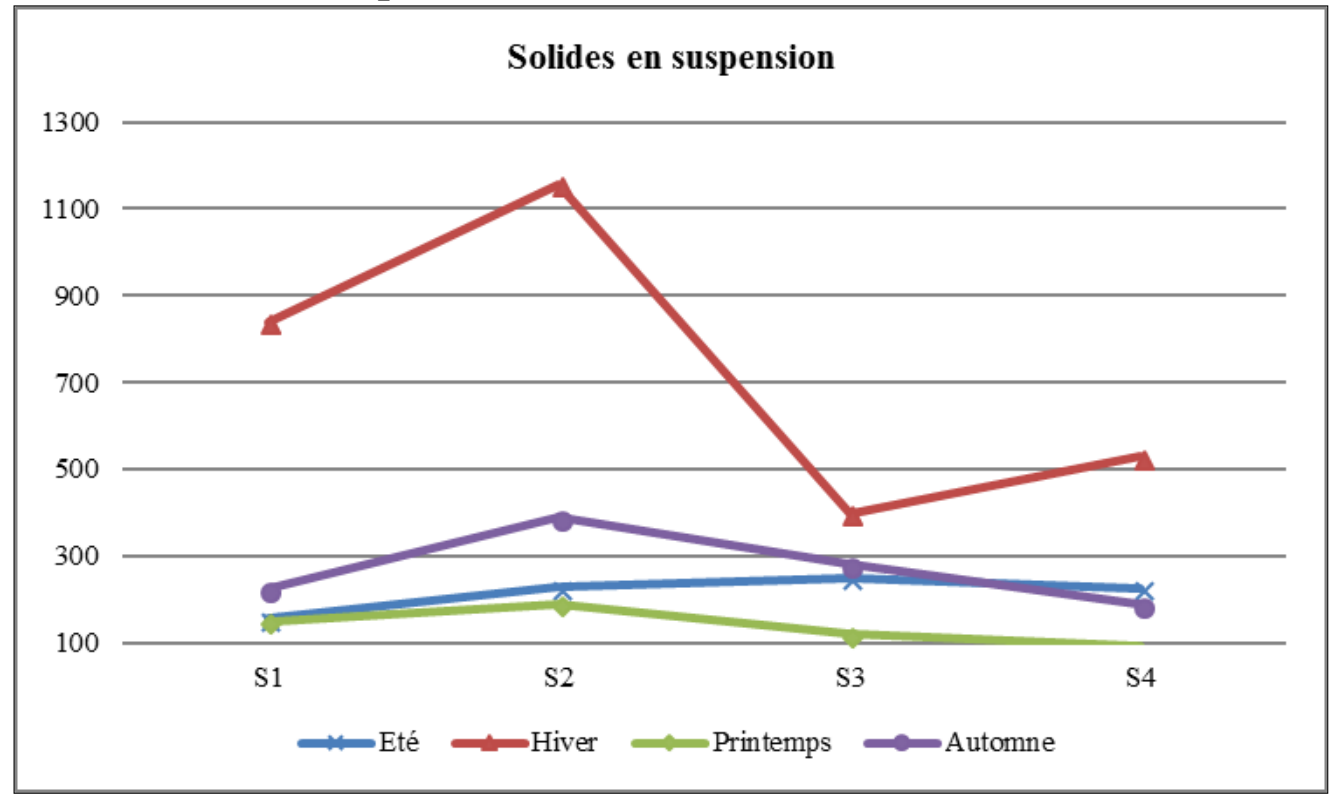

Figure 16 : Évolution spatio-temporelle et teneurs en matières en suspension

Les résultats des analyses (tableau 13) montrent que les concentrations minimales en MES ont été enregistrées en Printemps ( $95 \mathrm{mg} / \mathrm{l}$ à S4 et $150 \mathrm{mg} / 1$ à S1), tandis que les fortes teneurs ont été observées en hivers $(1157 \mathrm{mg} / \mathrm{l}$ en S2).

Le suivi saisonnier de MES (figure 10) montre que l'hiver marque des teneurs les plus élevées qui sont suivi de la période estivo-automnale. Ces fortes concentrations sont probablement le résultat de l'augmentation du débit de l'oued et des rejets important des margines dans ce dernier. Alors qu'en été et en automne, les valeurs enregistrées pourraient s'expliquer par la diminution du débit de l'oued et aux effluents des lixiviats issus de la décharge publique.

\section{Analyse explicative des paramètres physico-chimiques a- Analyse en composantes principale (ACP) des paramètres physico- chimiques \\ L'analyse spatiale des variables nous permet de distinguer entre deux groupes (fig.17) :}




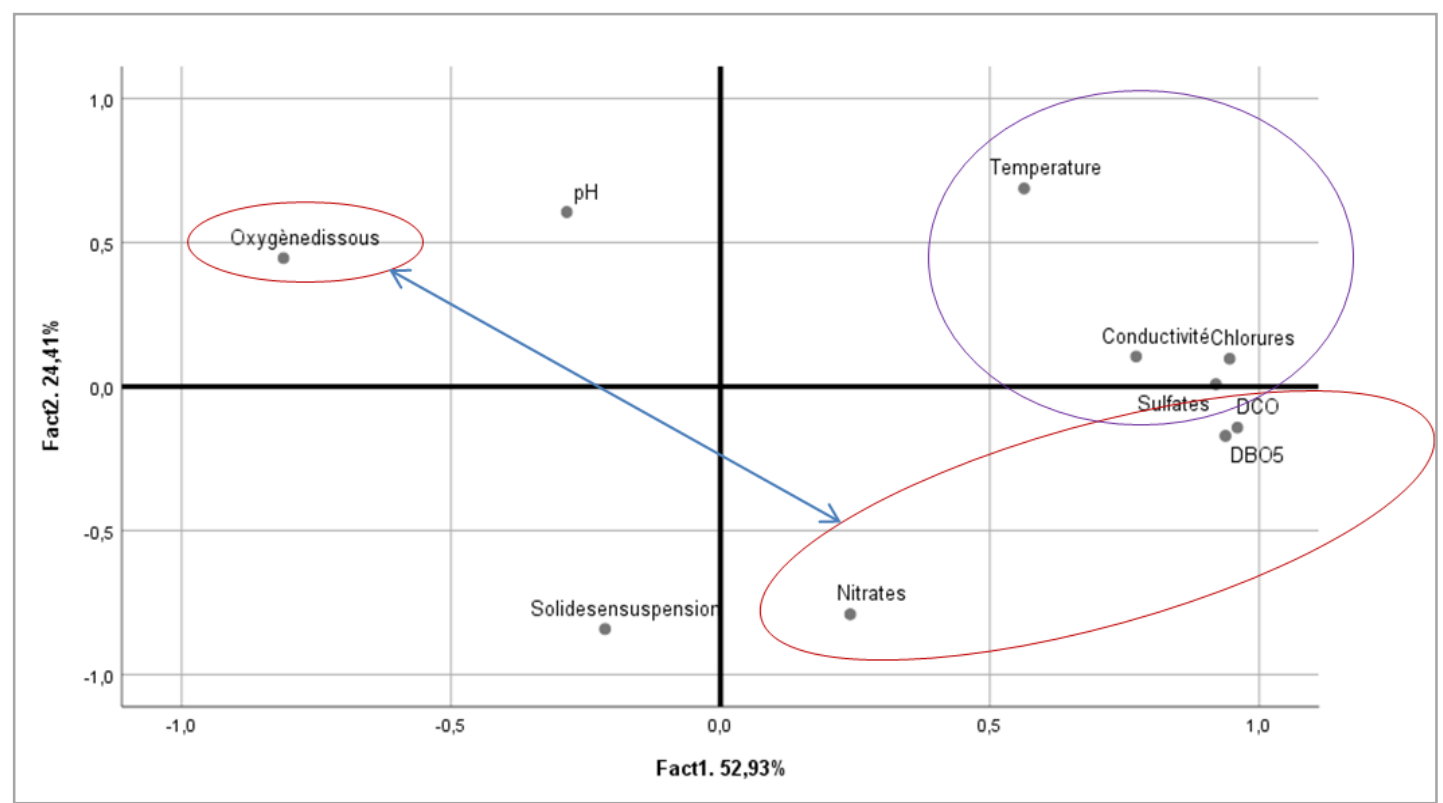

Figure 17 : Projection des variables sur le plan factoriel F1xF2

Groupe 1 : Il regroupe les paramètres : DCO, DBO5 et les nitrates qui sont fortement liés entre eux et corrélés positivement avec l'axe F1, et l'oxygène dissous qui y est associé négativement. Les valeurs élevées de la DCO et de la DBO5 pourrait s'expliquer par l'instauration des conditions de dégradation de la matière organique par les microorganismes dont l'activité et la multiplication, nécessitant l'oxygène, Pour les nitrates, la consommation de l'oxygène dissous est justifiée par la dénitrification des nitrites en nitrates par les bactéries autotrophes nitrosomonas et nitrobacters réalisant respectivement l'oxydation de l'ammonium en nitrite et l'oxydation des nitrites en nitrates. Ce groupe nous renseigne sur la majorité des paramètres qui déterminent le degré de la pollution organiques des eaux de l'Oued Boufekrane.

Groupe 2 : Il regroupe la conductivité, les chlorures, les sulfates et la température qui sont corrélée positivement avec l'axe F1. Ce groupe nous renseigne sur la pollution minérale.

Les matières en suspension sont corrélées négativement avec l'axe F1 et ne présentent aucune forte corrélation avec la DBO5 et la DCO ce qui nous amène à déduire que la matière en suspension sont liées au régime d'écoulement des eaux dans l'oued Boufekrane et non pas à l'impact des rejets des lixiviats de la décharge publique et des rejets des eaux usées traitées de la station d'épuration de Meknès.

L'analyse de la projection des individus sur le plan factoriel F1-F2 nous a permis de définir une typologie dominée par l'individualisation de deux groupes de stations (fig.18): 


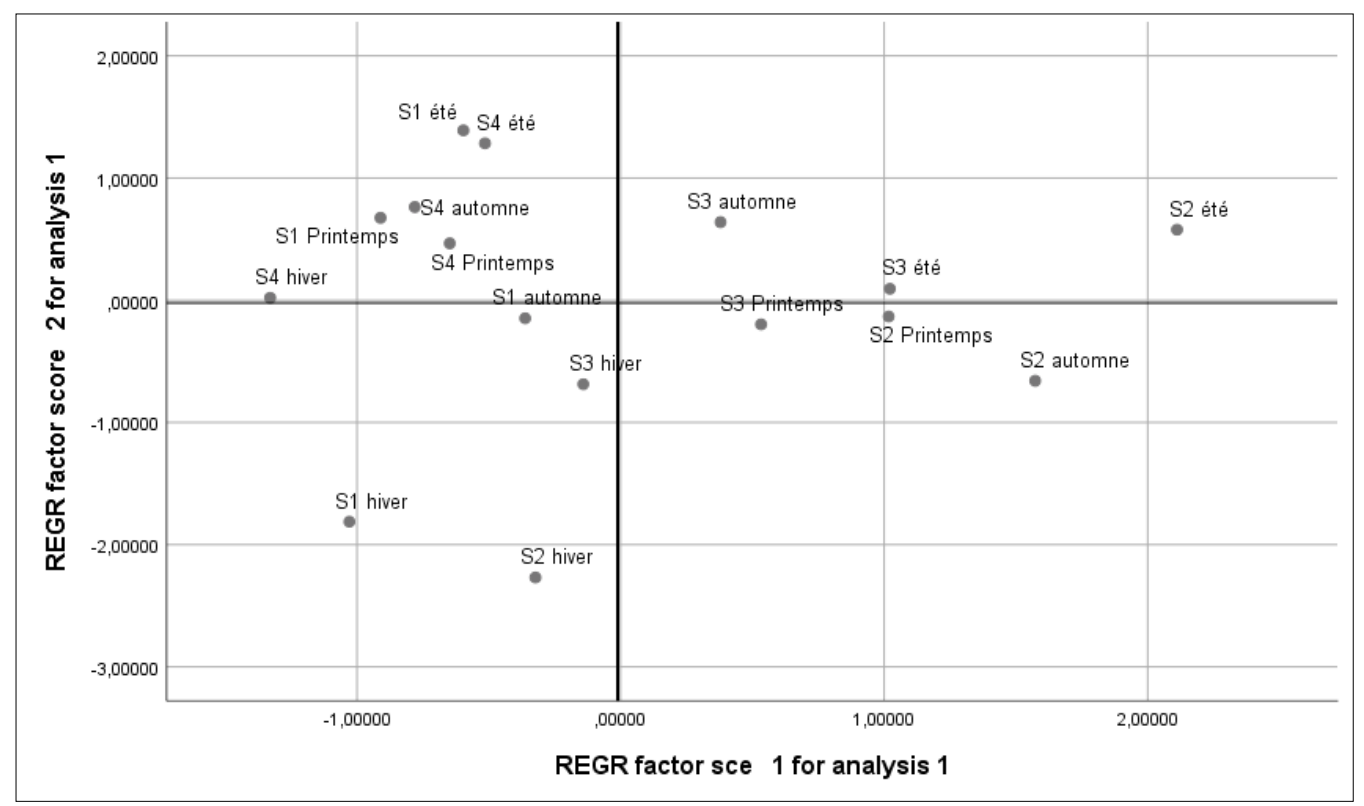

Figure 18: Projection des individus sur le plan factoriel F1xF2

Groupe 1: Ce groupe d'individus constitué par les stations S1 et S4 (S1 située en amant de la décharge et la STEP et S4 en aval juste avant la confluence avec l'oued Ouislane), présente une corrélation positive avec le $\mathrm{pH}$ et l'oxygène dissous sur l'axe F2 et négative avec la DCO et la DBO5. Les eaux de ce groupe sont donc moins polluées et sont caractérisées par des teneurs relativement faibles en matières organiques et relativement bien oxygénée.

Groupe 2 : ce groupe d'individus constitué par les stations (S2 et S3), présente une corrélation positive avec la $\mathrm{DCO}, \mathrm{DBO}$, conductivité, chlorures et sulfates sur l'axe F1. Les eaux de ce groupe correspondent aux eaux de l'oued les plus chargées en matière organique et minérale et les plus menacées par les rejets de la décharge et la STEP.

Sur la base des données trimestrielles récoltées, l'analyse en composante principale confirme que les résultats obtenus sont très cohérents et concorde avec l'analyse descriptive.

\section{Conclusion}

L'analyse physico chimique des eaux de l'Oued Boufekrane nous a montré la forte dégradation de la qualité de ces derniers (salinité élevée et fortes teneurs en matières organiques) due essentiellement à leur contamination par l'écoulement des lixiviats issus de la décharge publique de la ville. Par conséquent, il s'avère nécessaire de concevoir un réseau de collecte des lixiviats dans la perspective d'installation d'une station d'épuration. 


\section{References:}

1. Aboulkacem, A., Chahlaoui, A., Soulaymani, A., Rhazi-Filali, F., \& Benali, D. (2007). Etude comparative de la qualité bactériologique des eaux des oueds Boufekrane et Ouislane à la traversée de la ville de Meknès (Maroc). Rev. Microbiol. Ind. San. Environ, 2007, Vol 1, pp10-22.

2. Ahmamou, H. (1987). Etude sédimentologique des calcaires lacustres Saïssiens (Plio-Quaternaire) du bassin de Fès-Meknès (Maroc). Thèse de 3éme Cycle, Université Aix-en Provence, Marseille, 178 p, 1987.

3. Ait Brahim, L. (1991). Tectonique cassante récente au nord du Maroc. Contribution à l'étude du risque sismotectonique, Thèse de Doctorat, Université Mohamed V, Rabat, 223 p, 1991.

4. Amraoui, F. (2005). Contribution à la connaissance des aquifères karstiques : cas de Lias de la plaine de Saïss et du Causse moyen atlasique tabulaire (Maroc), Thèse de Doctorat, Université Hassan II Aïn Chock, Casablanca, 227 p, 2005.

5. Ansa-Asare, O. D., Marr, I. L., et Cresser, M. S. (1999). p Evaluation of cycling patterns of dissolved oxygen in a tropical lake as an indicator of biodegradable organic pollution. Science of the total environment, 1999, vol. 231, no 2-3, p. 145-158.

6. Benabdellouahad, S. (2006). Structure, dynamique et typologies physico-chimiques et phytoplanctoniques de l'estuaire du Bou Regreg (Côte Atlantique Marocaine).

7. Bouaouin, O, Khalil, F, Chtioui, H, Zaitan, H, Harrach, A. (2015). Traitement par électrocoagulation des lixiviats de la décharge publique contrôlée de la ville de Fès. Larhyss Journal, ISSN1112-3680, September, 53-67.

8. Boumir, K. (1987). Nouvelle interprétation du mode de mise en place des sables fauves dans le bassin du Saïss (Maroc). Thèse 3ème Cycle, Université Sidi Mohammed Ben Abdellah, Fès, Maroc, 169p, 1987.

9. Chofqi, A. Younsi, A. Lhadi, E. Mania, J. Mudry, J. Veron, A. (2003). Pollution d'une nappe phréatique par les métaux lourds du lixiviat de

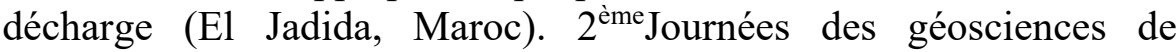
l'environnement, Université Ibn Tofail, Kenitra, Maroc, 2003.

10. Chiguer, H, EL Khayyat, F, EL Rhaouat, O, Rifki, R, Bensaid, A, EL Kharrim, K. Belghyti, D. (2016). Evaluation de la charge polluante des lixiviats de la décharge contrôlée de la ville d'Essaouira (MAROC), International Journal of Innovation and Applied Studies ISSN 20289324 Vol. 14 No. 3 Feb. 2016, pp. 863-874.

11. CID/SEURECA., (2006). Etude d'actualisation du schéma directeur d'assainissement liquide de l'agglomération de la ville de Meknès, 197 p, 2006. 
12. Dumontier, M., Dethier, M., Revaclier, R., \& Balikungeri, A. (1995). Etude hydrobiologique du bassin de l'Hermance. Publications de la Société Linnéenne de Lyon, Vol 64, num 09, pp 411-422.

13. El Addouli, J. (2010). Qualité physico-chimique et biologique des eaux polluées réutilisées en agriculture dans la zone périculture de Meknès (Maroc). Meknès (Maroc): Facultés des Sciences, Université Moulay Ismaïl.

14. Essahlaoui, A. (2000). Contribution a la reconnaissance des formations aquifères dans le bassin de Meknès Fès, prospection géoélectrique étude hydrogéologique et intervenir des ressources en eau, Thèse de Doctorat, Université Mohamed V, Rabat, 258 p, 2000.

15. Fassi, D. (1999). Les formations superficielles du Saïss de Fès et de Meknès. Du temps géologique à l'utilisation actuelle des sols, Notes et Mémoire de Service Géologique, 389, 524 p, 1999.

16. Harmouzi, O. (2010). Reconnaissance détaillée de la partie nord-est du Bassin de Saïss (MAROC): interprétation de sondages électriques verticaux par combinaison des méthodes statistique, géostatistique et d'inversion, thèse de doctorat, Université Moulay Ismail, Meknès, 304 p, 2010.

17. Hakkou, R. (2001). La décharge publique de Marrakech : caractérisation des lixiviats, étude de leur impact sur les ressources en eau et essai de leur traitement. Thèse de doctorat d'état, université de CAPI AYYAD, Maroc, 150p, 2001.

18. Karrouch, L. (2010). Bio-évaluation de la qualité des eaux courantes de la région Meknès (Centre-Sud, Maroc)-Impact sur l'environnement et la sante (Doctoral dissertation, Thèse doctorat es sciences en biologie. Université Moulay Ismaïl, Meknès (Maroc)).

19. Kouassi A.E. Ahoussi K.E. Koffi Y.B., Kouame I.K. Soro N. Biemi J. (2014). Caractérisation physico-chimique du lixiviat d'une décharge de l'Afrique de l'Ouest: Cas de la décharge D'Akouedo (Abidjan-Cote d'ivoire), Larhyss Journal, ISSN1112-3680, September, 63-74.

20. Larif, M., Soulaymani, A., Hnach, M., \& El Midaout, A. (2013). Contamination spatio-temporelle d'origine hydrique de l'oued Boufekrane dans la région de Meknès-Tafilalt (Maroc). International Journal of Biological and Chemical Sciences, Vol 07, $\mathrm{n}^{\circ}$ 01, pp 172184.

21. Mejraoua, Z. Zine, N. (2017). Caractérisation physico-chimique du Lixiviat

de la décharge sauvage de Meknès, Européen Scientific Journal November édition vol.13, No.33 ISSN : 1857 - 7881 (Print) e - ISSN 1857- 7431. 
22. Neal, C., \& Robson, A. J. (2000). A summary of river water quality data collected within the Land-Ocean Interaction Study: core data for eastern UK rivers draining to the North Sea. Science of the Total Environment, Vol 251, pp 585-665.

23. Ourghache, D. (1987). Etude géologique dans le Paléozoïque et le Trias de la bordure NW du causse moyen atlasique ( $\mathrm{S}$ et SW de Fès, Maroc), thèse de 3éme cycle, Université de Paul Sabatier, Toulouse, 130 p, 1987.

24. Rodier J., Legube B., Merlet N., Coll. (2009). L'analyse de l'eau, 9ème édition. Ed. Dunod Paris. ISBN: 978- 2-10-054179-9

25. Sita. (2013). Etude d'impact sur l'environnement de la création et l'exploitation du centre d'élimination et de valorisation des déchets de la ville de Meknès, 150p, 2013.

26. Site internet :

sa.com/environnement/decharges.htm

http://www.diastrata-

27. Taltasse, P. (1953). Recherches géologiques et hydrogéologiques dans le bassin lacustre de Fès-Meknès. Notes et Mémoire de Service Géologique, 115, 300 p, 1953. 